Revista Brasil. Bot., V.32, n.1, p.33-55, jan.-mar. 2009

\title{
Palinotaxonomia das espécies de Cordiaceae (Boraginales) ocorrentes no Estado de São Paulo ${ }^{1}$
}

\author{
EDUARDO CUSTÓDIO GASPARINO ${ }^{2,3}$ e MARIA AMÉLIA VITORINO DA CRUZ BARROS²
}

(recebido: 28 de fevereiro de 2008; aceito: 07 de novembro de 2008)

\begin{abstract}
Palynotaxonomy of Cordiaceae (Boraginales) from São Paulo State). The pollen morphology of 18 heterostylic species, representing 2 genera, of Cordiaceae from the state of São Paulo were investigated. The pollen grains were acetolyzed, measured, described and photomicrographed under light microscope, and when necessary, under scanning electron microscope. Cordia has 3-colporate pollen grains with long colpus and lalongate endoaperture, the exine is spiny to spinulate-verrucate. Varronia has 3-porate pollen grains and pores with operculum, the exine is reticulated, homobrochate to heterobrochate. In most of the species the pollen grains of the long-styled (pin) flowers have higher diameter values. Although Cordia and Varronia can be easily distinguished based on pollen morphology, both genera are stenopalynous and it is not possible to distinguish species based on pollen grains morphology, though different group of species have pollen grains with different average size, size measures overlap.
\end{abstract}

Key words - Boraginaceae, Cordiaceae, heterostyly, pollen grains

RESUMO - (Palinotaxonomia das espécies de Cordiaceae (Boraginales) ocorrentes no Estado de São Paulo). É apresentado o estudo polínico das 18 espécies heterostílicas de Cordiaceae, representando dois gêneros, ocorrentes no Estado de São Paulo, visando interpretar a morfologia polínica e fornecer dados para a taxonomia da família. Os grãos de pólen foram acetolisados, medidos, descritos e fotomicrografados em microscopia óptica. Para observar detalhes da ornamentação, grãos de pólen não acetolisados foram analisados em microscopia eletrônica de varredura (MEV). A morfologia polínica revelou dois tipos polínicos: Cordia com grãos de pólen 3-colporados, colpos longos e endoaberturas lalongadas, exina espinhosa a espículo-verrugosa e Varronia com grãos de pólen 3-porados, poros com opérculos, exina reticulada, homorreticulada a heterorreticulada. Na maioria das espécies estudadas, os grãos de pólen das flores longistilas apresentaram valores maiores de diâmetros. Os dados obtidos mostram Cordiaceae como euripolínica, enquanto que os gêneros Cordia L. e Varronia P. Br. são estenopolínicos.

Palavras-chave - Boraginaceae, Cordiaceae, grãos de pólen, heterostilia

\section{Introdução}

A família Boraginaceae é tradicionalmente dividida em quatro subfamílias: Ehretioideae, Cordioideae, Helitropioideae e Boraginoideae (Johnston 1950, Nowicke \& Miller 1990, Miller 2007). Porém, estudos moleculares recentes evidenciaram a elevação destas subfamílias ao nível de famílias (Gottschling et al. 2001, 2005, Miller 2007, Miller \& Gottschling 2007).

Cordiaceae é considerada uma família monofilética, cosmopolita e provavelmente a mais complexa de Boraginales (Nowicke \& Ridgway 1973, Taroda \& Gibbs 1986, Miller \& Gottschling 2007). Possui aproximadamente 350 espécies (Judd et al. 1999, Miller 2001), com grande diversidade no Novo Mundo e cerca de 65 espécies no

\footnotetext{
1. Parte da dissertação de mestrado do primeiro autor, Programa de Pós Graduação em Biodiversidade Vegetal e Meio Ambiente, Instituto de Botânica, São Paulo, SP, Brasil.

2. Instituto de Botânica, Seção de Dicotiledôneas, Caixa Postal 3005, 01061-970 São Paulo, SP, Brasil.

3. Autor para correspondência: ebotanic@yahoo.com.br
}

Brasil (Taroda \& Gibbs 1986). Atualmente a família abrange os gêneros Coldenia L., Cordia L. e Varronia P. Br., sendo nativas no Brasil somente espécies de Cordia L. e Varronia P. Br. (Barroso et al. 1986, Cavalheiro et al. 2003, Miller \& Gottschling 2007).

A heterostilia, característica bem conhecida nas espécies de Cordiaceae (Tomlinson 1974, Opler et al. 1975, Ganders 1979, Gibbs \& Taroda 1983) é um polimorfismo floral controlado geneticamente no qual as flores diferem reciprocamente nos comprimentos de estiletes e estames (Ganders 1979). Este polimorfismo floral é geralmente acompanhado de um sistema de autoincompatibilidade que impede a autofecundação (Barret 1990, Gibbs 1990, Jung-Mendaçolli \& Melhem 1995) e pode ser representado por duas ou três formas florais (distilia e tristilia), havendo, às vezes, diferenças no tamanho e quantidade de grãos de pólen produzidos pelas diferentes formas florais, assim como na ornamentação da exina dos grãos de pólen, nas papilas estigmáticas e no tamanho e morfologia da corola (Ganders 1979).

A partir do trabalho de Erdtman (1952), foram realizados outros estudos sobre a morfologia polínica de 
algumas espécies da família, destacando-se os trabalhos de Nowicke \& Ridgway (1973), Taroda (1984), PaláciosChávez \& Quiroz-Garcia (1985), Moncada \& Herrera (1988), Nowicke \& Miller (1990) e Scheel et al. (1996).

Tendo em vista que os trabalhos envolvendo a morfologia polínica de Cordiaceae sempre estão relacionados com espécies isoladas e raramente comparam de forma criteriosa os grãos de pólen das flores brevistilas e longistilas, o objetivo deste trabalho foi estudar a morfologia polínica de 18 espécies da família listadas como nativas para o estado de São Paulo (N. Taroda-Ranga, dados não publicados), fornecendo assim, dados para a sua taxonomia, além de verificar as diferenças quantitativas e qualitativas entre os grãos de pólen das espécies heterostílicas.

\section{Material e métodos}

Os materiais utilizados para as preparações palinológicas foram obtidos a partir de exsicatas dos herbários BOTU, ESA, HRCB, IBGE, R, RB, SJRP, SP, SPF, SPSF, UEC, cujos acrônimos seguem Holmgren \& Holmgren (1998).

Foram estudadas separadamente as formas brevistila e longistila. Para cada espécie e forma determinou-se um espécime como "padrão", que é citado abaixo seguido de asterisco (*) e que foi utilizado para as descrições e ilustrações polínicas. Procurou-se, sempre que possível, estudar outros espécimes de cada espécie, denominados "material de comparação". As lâminas com o material polínico encontramse depositadas na palinoteca da seção de Dicotiledôneas no Instituto de Botânica de São Paulo. Devido à escassez de materiais coletados no Estado de São Paulo, algumas vezes foram examinadas coletas provenientes de outros estados.

Cordia ecalyculata Vell.: Brevistila: BRASIL. MINAS GeraIs: Juiz de Fora, Fazenda do Sertão, 25-XI-1946, E.P. Heringer 1117 (SP)*. Rio GRANDE Do Sul: Itapuã, Viamão, 13-I-1985, M. Sobral 3688 (SP). SÃo Paulo: Ribeirão Preto, Fazenda Baixadão, 24-VII-1998, M.A. Assis et al. 1236 (HRCB); São Paulo, Butantã, I-1921, A. Gehrt s.n. (SP5304); Sapopema, Salto das Orquídeas, 8-XI-1997, C. Medri et al. 501 (HRCB). Longistila: Paraná: Cambé, Parque Municipal Peroba Rosa, 7-XI-1997, V.F. Кinupp \& E.M. Francisco 946 (UEC). São Paulo: Mogi-Guaçu, Estação Experimental, 20X-1977, P.E. Gibbs \& H.F. Leitão Filho 6093 (UEC); Rio Claro, Jardim da Universidade Estadual Paulista, 23-XI-1984, A. Furlan 207 (HRCB); idem, Sítio Porteira, 12-XI-1987, C.M. Beltrati 113 (HRCB)*. C. glabrata (Mart.) DC.: Brevistila: BRASIL. GoIÁs: Padre Bernardes, 11-VIII-1974, E.P.Heringer \& Paula 13922 (UEC). Minas GERAIS: Ituiutaba, Cachoeira Dourada, 16-VI-1946, A. Macedo 775 (SP)*. São Paulo: Campinas, Fazenda Santa Elisa, 11-VIII-1983, J.R. Trigo 15121 (UEC). Longistila: GoIÁs: Piranhas, 27-VII-1977, G. Hatschbach 40134 (UEC). Mato Grosso: Santo Antonio de Leverger, Fazenda Experimental da Universidade Federal do Mato Grosso, 28-VIII-1996, H.B.N. Borges 35469 (UEC). São Paulo: Campinas, Instituto Agronômico, 4-IX-1978, H.M. Souza 8354 (UEC); idem, Bosque das Palmeiras CATIIAC, 4-IX-1991, H. Lorenzi s.n. (SP262131); idem, Parque Ecológico da Universidade Estadual de Campinas, 27-VII1993, D.A. Santin 309915 (UEC)*. C. magnoliifolia Cham.: Brevistila: BRASIL. São PAULO: São Paulo, Butantã, 10XII-1924, A. Gehrt et al. s.n. (SP18135)*; idem, Alto da Lapa, 15-IV-1946, W. Hoehne s.n. (SPF11629). Longistila: RIO DE Janeiro: Petrópolis, Caetitri, II-1944, O.C. Góes \& D. Constantino 143 (RB)*. C. rufescens A. DC.: Brevistila: BRASIL. São PaUlo: Itapetininga, XII-1943, J.I. Lima s.n. (RB48958)*. Longistila: Paraná: Quatinguá, Rodovia BR 92, 19-XI-1976, G. Hatschbach 39243 (SP)*; Venceslau Brás, estrada para Arapoti, 3-XII-1988, A.O.S. Vieira et al. 240 (UEC). C. sellowiana Cham.: Brevistila: BRASIL. PARANÁ: Paranaguá, Morro do Joaquim, 9-XI-1985, R.M. Britez 24695 (UEC). SÃo Paulo: Atibaia, Pico da Pedra Grande, 7-VI-1988, A.A. Meira Neto et al. 21515 (SJRP); Birigui, Fazenda Água-Branca, 27-VII-2000, E. Montilha et al. s.n. (SJRP22970)*; Campinas, Praça João Lech Júnior, 18-VIII2000, R. Cielo Filho 184 (UEC); Pariquera-Açu, Estação Experimental do Instituto Agronômico, 19-IV-1995, N.M. Ivanauskas 148 (SJRP); idem, 18-I-1996, N.M. Ivanauskas 676 (SJRP). Longistila: São PAULo: Birigui, Fazenda ÁguaBranca, 11-VIII-1999, E. Montilha et al. s.n. (SJRP20061)*. C. silvestris Fresen.: Brevistila: BRASIL. SÃo PaUlo: Ubatuba, Picinguaba, 6-I-1993, M.A. Assis 44 (HRCB); idem, Instituto Agronômico de Campinas, 1-II-1996, H.F. Leitão Filho et al. s.n. (SP295159)*; idem, Estação Experimental, 10-I-1979, A.F. Silva 10995 (UEC). Longistila: PARANá: Morretes, Estrada da Graciosa, 30-I-1974, G. Hatschbach 33779 (UEC)*. C. superba Cham.: Brevistila: BRASIL. São PAUlo: MogiGuaçu, Estação Experimental, 30-IV-1996, G. Marinis 545 (HRCB); Pindamonhangaba, 14-IV-1934, E. Amaral s.n. (SP31708)*. Longistila: SÃo PAULO: Guaratinguetá, Reserva Florestal da Escola Estadual de Especialistas da Aeronáutica, X-2000, D.C. Cavalcanti 261 (HRCB); Itapetininga, Campo D. Pickel, 12-XI-1948, A. Jordão s.n. (SPSF3336)*; MogiGuaçu, Fazenda Campininha, 20-X-1989, O. César et al. s.n. (HRCB11286); idem, mata ciliar, 3-III-1993, J.V. Godoi et al. 283 (SP); Rio Claro, Universidade Estadual Paulista, 20-XI-1984, A. Furlan 201 (HRCB); Taguaí, estrada para Itaporanga, 28-IX-1994, J.Y. Tamashiro et al. 685 (UEC). C. taguahyensis Vell.: Brevistila: BRASIL. São PAUlo: Caraguatatuba, Parque Estadual, 17-I-1990, M. Imannoto s.n. (SPSF13297)*. Longistila: EspíRIto SANTO: Linhares, norte do Rio Doce, 22-II-1988, W.W. Thomas et al. s.n. (SPF63507)*. São PAUlo: Ubatuba, Estação Experimental, 1-IX-1939, A. Gehrt s.n. (SP44380); idem, Ilha Anchieta, 13-XII-1999, H.F. Leitão Filho et al. 32579 (UEC). C. trichotoma (Vell.) Arrab. ex Steud.: Brevistila: BRASIL. São Paulo: Birigui, Fazenda Água-Branca, 26-V-1999, E. Montilha et al. s.n. (SJRP19921); Cruzeiro, estrada da Fazenda Boa Vista, 5-IV-1995, G.J. Shepherd \& I. Koch 14 (SP); Gália, Estação Ecológica dos Caetetus, 29-VI-1995, 
F.C. Passos \& A.C. Kim 37 (UEC); Jundiaí, Fazenda Malota, 9-V-1977, N.H. Traldi \& S.T. Souza 25 (UEC); Potirendaba, mata ciliar do Ribeirão Cubatão, 30-IV-1999, J.C. Souza et al. 32 (SJRP); Teodoro Sampaio, Parque Estadual do Morro do Diabo, 16-V-1995, M. Kirizawa et al. 3077 (SP)*; Votuporanga, fazenda vizinha da Estação Experimental do IAC, 16-V-1995, L.C. Bernacci et al. 1638 (SJRP). Longistila: SÃo PAUlo: Campinas, Distrito de Barão Geraldo, 14-VI-1977, P.E. Gibbs 4781 (UEC); idem, Parque Ecológico da Universidade Estadual de Campinas, 27-V1993, D.A. Santin 30925 (UEC); Nazaré Paulista, estrada de terra para Piracaia, 10-IV-1995, J.Y. Tamashiro et al. 772 (SP)*. Varronia calocephala (Cham.) Fresen: Brevistila: BRASIL. Goiás: Alto do Paraíso, Chapada dos Veadeiros, 18-III-1976, J. Semir s.n. (UEC993)*. Longistila: GoIÁs: Serra Dourada, I-1893, G.M. Ulle $677(\mathrm{R})^{*}$. V. curassavica Jacq.: Brevistila: BRASIL. SÃo PAULO: Campinas, altura do km 139 da estrada D. Pedro I, 5-X-1992, A.P. Spina 38 (SJRP); idem, Barão Geraldo, 16-II-1995, A.P. Spina 391 (UEC); Ilha Comprida, Pedrinhas, 23-VI-1999, N. Hanazaki et al. 192 (UEC)*; Zacarias, mata à beira da estrada, 8-X1999, N. Taroda s.n. (SJRP20333). Longistila: SÃo PAulo: Campinas, Barão Geraldo, 26-X-1994, A.P. Spina 348 (UEC); Cananéia, Ilha Comprida, 8-IX-1994, J.R.R. Hoffmann et al. 62 (HRCB); idem, Ilha Comprida, 8-IX1994, J.R.R. Hoffmann et al. 66 (HRCB); Ubatuba, Parque Estadual da Serra do Mar, Núcleo Curucutu, 14-IV-2001, J.P. Souza et al. 3584 (UEC); idem, Praia do Puruba, 10XI-1993, A.P. Spina et al. 29156 (UEC)*; idem, Praia do Tenório e Praia do Cedro, 4-II-1996, H.F. Leitão Filho et al. 34652 (UEC). V. discolor (Cham.) Borhidi: Brevistila: BRASIL. Distrito Federal: Reserva Ecológica do IBGE, 18-XII-1995, M.A. Silva 2845 (IBGE). São Paulo: Avaí, Aldeia Guarani, 4-II-1999, A.P. Bertoncini \& A. Cazetta Neto 978 (SJRP); Pariquera-Açu, 22-XI-1995, N.M. Ivanauskas 571 (SJRP)*; São José do Rio Preto, Bosque Municipal, 30-III-1995, K.G. Melzi \& E.R. Avelisso s.n. (SJRP13926). Longistila: SÃo PAulo: Sete Barras, Barras de São Miguel, 13-II-1995, H.F. Leitão Filho et al. s.n. (SP278122)*. V. guazumaefolia Desv.: Brevistila: BRASIL. Goiás: Aporé, margem da estrada km 72, 2-IX-1995, M.R. Pietrobom da Silva et al. 2412 (SJRP)*. Longistila: São Paulo: Cajuru, Fazenda Santa Carlota, 24-IX-1989, A. Sciamarelli \& J. Vicente 264 (UEC)*. V. leucocephala (Moric.) J.S. Miller: Brevistila: BRASIL. Pernambuco: Sertânia, vegetação de caatinga, 22-XI-1999, L.S. Figueiredo 543 (SJRP)*. Longistila: B AHIA: Montes Claros, margem da estrada BR-262 para Aracatu, 12-XII-1984, G.P. Lewis et al. s.n. (SPF36329)*. V. monosperma Jacq.: Brevistila: BRASIL. São Paulo: Campinas, Mata Santa Genebra, 14IX-1989, L.C. Bernacci 34149 (UEC); idem, espontânea no campo, 25-IX-2001, G. Figueira \& V.C. Souza et al. s.n. (SJRP25847); Itararé, Estrada de Itararé-Bom Sucesso, 11XII-1997, F. Chung et al. 53 (UEC)*; Ubatuba, Trilha da Almada, 27-VIII-1994, M.A. Assis et al. 300 (HRCB). Longistila: São Paulo: Socorro, Bairro dos Domingues, 7-
III-2000, M. Groppo Júnior. 375 (SPF)*. V. sessilifolia (Cham.) Borhidi: Brevistila: BRASIL. Minas Gerais: Formosa, Parque Nacional Grande Sertão-Veredas, 1-XII1997, R.C. Mendonça et al. 3329 (RB)*; s.m., X-1862, Lad Neto 217 (R). São Paulo: São Paulo, 30-X-1887, A. Loefgren 289 (R). Longistila: BRASIL. BAHIA: Pico das Almas, 1300 m altitude, 19-II-1987, R.M. Harley et al. s.n. (SPF49775)*. V. truncata (Fresen.) Borhidi: Brevistila: BRASIL. Distrito FEDERAL: Reserva Ecológica do IBGE, 28-I-1987, M.A. Silva et al. 347 (IBGE)*. Longistila: Distrito Federal: Fazenda Água Limpa, Universidade de Brasília, 9-I-1990, D. Alvarenga \& F.C.A. Oliveira 585 (IBGE)*. V. urticifolia (Cham.) J.S. Miller: Brevistila: BRASIL. São PAulo: Ilha Bela, XI-1990, V.C. Souza 9472 (SJRP); Nazaré Paulista, Represa Atibainha, 1-VI-1996, V.C. Souza et al. 11203 (ESA); Ubatuba, Estação Experimental, 24-XI-1938, A.S. Costa \& I. Ramos et al. s.n. (SPSF4421)*. Longistila: SÃo Paulo: Campinas, Pasto Possi \& Borghi, 12-VI-1936, J. Santoro s.n. (SPSF637)*.

Para a análise em microscopia óptica, foi utilizado o método de acetólise de Erdtman modificado (Melhem et al. 2003). Os grãos de pólen foram medidos dentro do prazo máximo de uma semana (Melhem \& Matos 1972, SalgadoLabouriau 1973). No material padrão foram medidos os diâmetros, em 25 grãos de pólen acetolizados, tomados ao acaso e distribuídos em pelo menos três lâminas (SalgadoLabouriau et al. 1965). Para outros caracteres como aberturas, espessura das camadas da exina, espinhos e tamanho dos lumens foram feitas sempre que possível 10 medidas, o mesmo ocorrendo para as medidas dos diâmetros dos grãos de pólen dos materiais de comparação. Foram realizados tratamentos estatísticos adequados ao tamanho das amostras. Para comparar as médias duas a duas utilizouse o intervalo de confiança (IC) a 95\% (Vieira 1981, Zar 1984), cujos dados foram representados em gráficos usando o pacote estatístico MINITAB 10.3 for Windows (2003). Com objetivo de ordenar as espécies com características morfológicas polínicas semelhantes, foi realizada uma análise de componentes principais (PCA) nos grãos de pólen 3-colporados e 3-porados, baseada em 10 e nove variáveis métricas respectivamente (tabela 1), utilizando-se o programa FITOPAC (Shepherd 1996) e o programa PC-ORD for Windows versão 4.0 (McCune \& Mefford 1999).

Para a análise dos grãos de pólen em microscopia eletrônica de varredura (MEV) foi utilizada a metodologia descrita em Melhem et al. (2003), para grãos de pólen nãoacetolisados.

As fotomicrografias foram realizadas com auxílio de um microscópio Olympus BX50, com câmara de vídeo acoplada (SONY) e um microcomputador PC, sendo a captura e o tratamento das imagens feitos no programa Image Pro-Plus versão 3.0 para Windows. Já as eletromicrografias foram obtidas através de um microscópio PHILIPS XL Series XL 20, S/W, ver. 5.21.

A terminologia seguiu Barth \& Melhem (1988) e Punt et al. (2007). 
Tabela 1. Variáveis métricas dos grãos de pólen utilizadas na ordenação pela PCA (+ = presente; $-=$ ausente).

Table 1. Pollen grain metric variables used in PCA ordenation (+ = present; $-=$ absent).

\begin{tabular}{llcc}
\hline \multirow{2}{*}{ Variáveis } & & \multicolumn{2}{c}{ Grãos de pólen } \\
\cline { 3 - 4 } CCOM & (comprimento do colpo) & + & 3-porados \\
CLAR & (largura do colpo) & + & - \\
DPVE & (diâmetro polar em vista equatorial) & + & - \\
DEVE & (diâmetro equatorial em vista equatorial) & + & + \\
DEVP & (diâmetro equatorial em vista polar) & + & + \\
ESPI & (altura dos espinhos) & + & + \\
EXIN & (espessura da exina) & + & + \\
NEXI & (espessura da nexina) & + & + \\
PCOM & (comprimento do poro) & - & + \\
PLAR & (largura do poro) & + & + \\
SEXI & (espessura da sexina) & + & + \\
TETO & (espessura do teto) & & + \\
\hline
\end{tabular}

\section{Resultados}

Com base no tipo de abertura, as 18 espécies de Cordiaceae (figuras 1-60) aqui analisadas foram divididas em dois tipos polínicos:

Tipo I - Grãos de pólen 3-colporados (Cordia ecalyculata, C. glabrata, C. magnoliifolia, C. rufescens, C. sellowiana, C. sivestris, C. superba, C. taguahyensis e C. trichotoma)
- Grãos de pólen médios a grandes (tabela 2); isopolares; âmbito circular, subcircular a subtriangular (figura 14); suboblatos a oblato-esferoidais (tabela 3$) ; 3(-4)$ colporados; exina tectado-perfurada (figuras 10, 17, 28), sexina espinhosa a espículo-verrugosa ( $C$. superba figuras 23), espinhos supratectais com ápice agudo.

Os colpos são longos ou curtos (C. silvestris longistila), estreitos, com margem (C. glabrata e C. trichotoma) ou não; endoaberturas lalongadas (figuras 5, 29),

Tabela 2. Medidas dos grãos de pólen 3-colporados de espécies de Cordiaceae, em vista equatorial e polar $(n=25)$. (VE $=$ vista equatorial; $\mathrm{VP}=$ vista polar; $\mathrm{B}=$ brevistila; $\mathrm{L}=$ longistila; $x=$ média aritmética; $s_{x}=$ desvio padrão da média; $s=$ desvio padrão da amostra; $\mathrm{V}=$ coeficiente de variabilidade; $\mathrm{IC}=$ intervalo de confiança).

Table 2. Measures of 3-colporate pollen grains of Cordiaceae, in equatorial and polar views $(n=25)$. (VE $=$ equatorial view; $\mathrm{VP}=$ polar view $; \mathrm{B}=$ shortsyle $; \mathrm{L}=$ longstyle $; x=$ arithmetic mean; $s_{x}=$ medium standard deviation; $s=$ sample standard deviation; $\mathrm{V}=$ coefficient of variability; I.C. = confidence interval).

\begin{tabular}{cccccc}
\hline Espécies de Cordia & $\begin{array}{c}\text { Faixa de variação } \\
(\mu \mathrm{m})\end{array}$ & $\begin{array}{c}x \pm s_{x} \\
(\mu \mathrm{m})\end{array}$ & $\begin{array}{c}s \\
(\mu \mathrm{m})\end{array}$ & $\begin{array}{c}\text { V } \\
(\%)\end{array}$ & $\begin{array}{c}\text { I.C. } \\
(\mu \mathrm{m})\end{array}$ \\
\hline
\end{tabular}

Diâmetro polar (VE)

$\begin{array}{lllllr}\text { C. } \text { ecalyculata (B) } & 36,88-43,75 & 39,92 \pm 0,35 & 1,75 & 4,40 & 39,20-40,64 \\ \text { C. } \text { ecalyculata (L) } & 35,13-47,63 & 40,51 \pm 0,59 & 2,97 & 7,33 & 39,29-41,73 \\ \text { C. } \text { glabrata (B) } & 39,13-48,88 & 43,31 \pm 0,53 & 2,64 & 6,09 & 42,22-44,40 \\ \text { C. } \text { glabrata (L) } & 38,75-47,25 & 43,19 \pm 0,46 & 2,32 & 5,37 & 42,24-44,14 \\ \text { C. magnolifolia (B) } & 41,63-47,63 & 44,68 \pm 0,37 & 1,84 & 4,12 & 43,92-45,44 \\ \text { C. magnolifolia (L) } & 46,75-57,50 & 50,96 \pm 0,57 & 2,83 & 5,56 & 49,79-52,13 \\ \text { C. rufescens (B) } & 62,50-77,75 & 68,95 \pm 1,22 & 4,89 & 7,10 & 66,44-71,76 \\ & & & & & \text { continua }\end{array}$


continuação

\begin{tabular}{|c|c|c|c|c|c|}
\hline Espécies de Cordia & $\begin{array}{l}\text { Faixa de variação } \\
\qquad(\mu \mathrm{m})\end{array}$ & $\begin{array}{l}x \pm s_{x} \\
(\mu \mathrm{m})\end{array}$ & $\begin{array}{c}s \\
(\mu \mathrm{m})\end{array}$ & $\begin{array}{l}\mathrm{V} \\
(\%)\end{array}$ & $\begin{array}{l}\text { I.C. } \\
(\mu \mathrm{m})\end{array}$ \\
\hline C. rufescens $(\mathrm{L})$ & $65,88-88,63$ & $77,92 \pm 1,45$ & 7,26 & 9,32 & $74,93-80,91$ \\
\hline C. sellowiana (B) & $29,13-36,13$ & $32,20 \pm 0,34$ & 1,70 & 5,28 & $31,50-32,90$ \\
\hline C. sellowiana (L) & $29,88-36,38$ & $32,19 \pm 0,30$ & 1,55 & 4,82 & $31,57-32,81$ \\
\hline C. silvestris (B) & $37,50-49,00$ & $44,10 \pm 0,41$ & 2,06 & 4,67 & $43,26-44,94$ \\
\hline C. silvestris (L) & $37,50-43,25$ & $39,40 \pm 0,28$ & 1,42 & 3,61 & $38,82-39,98$ \\
\hline C. superba (B) & $66,25-80,38$ & $72,61 \pm 0,75$ & 3,74 & 5,15 & $71,06-74,16$ \\
\hline C. superba (L) & $67,25-78,00$ & $71,26 \pm 0,63$ & 3,27 & 4,59 & $69,96-72,56$ \\
\hline C. taguahyensis (B) & $66,75-78,88$ & $71,59 \pm 0,71$ & 3,56 & 4,97 & $70,13-73,05$ \\
\hline C. taguahyensis (L) & $59,75-75,00$ & $66,61 \pm 0,80$ & 4,00 & 6,00 & $64,96-68,26$ \\
\hline C. trichotoma $(\mathrm{B})$ & $34,50-43,50$ & $39,31 \pm 0,46$ & 2,26 & 5,75 & $38,36-40,26$ \\
\hline C. trichotoma $(\mathrm{L})$ & $33,13-42,88$ & $37,63 \pm 0,56$ & 2,72 & 7,23 & $36,48-38,78$ \\
\hline
\end{tabular}

C. ecalyculata (B)

C. ecalyculata (L)

C. glabrata (B)

C. glabrata (L)

C. magnolifolia (B)

C. magnolifolia (L)

C. rufescens (B)

C. rufescens (L)

C. sellowiana (B)

C. sellowiana $(\mathrm{L})$

C. silvestris (B)

C. silvestris (L)

C. superba (B)

C. superba (L)

C. taguahyensis (B)

C. taguahyensis (L)

C. trichotoma (B)

C. trichotoma (L)

C. ecalyculata (B)

C. ecalyculata (L)

C. glabrata (B)

C. glabrata (L)

C. magnolifolia (B)

C. magnolifolia (L)

C. rufescens (B)

C. rufescens (L)

C. sellowiana (B)

C. sellowiana $(\mathrm{L})$

C. silvestris (B)

C. silvestris (L)

C. superba (B)

C. superba (L)

C. taguahyensis (B)

C. taguahyensis (L)

C. trichotoma (B)

C. trichotoma (L)
$42,13-49,25$

42,50-52,13

44,25-54,63

41,38-48,63

46,13-56,25

$55,00-64,88$

60,00-90,50

$79,50-97,50$

$33,38-41,75$

34,13-42,00

44,25-52,50

$41,63-48,25$

$80,38-90,00$

$77,63-87,13$

$70,38-86,50$

68,63-82,88

$35,88-45,63$

$36,13-43,88$

$41,13-48,50$

41,38-50,00

42,00-50,63

40,50-48, 25

41,13-51,75

49,25-60,38

$72,38-89,88$

78, 13-98,63

$32,63-40,50$

$33,75-40,25$

$43,63-50,25$

40,75-45,88

$75,00-84,75$

$72,38-87,13$

$61,38-86,75$

$62,50-84,88$

40,13-50,00

$36,50-43,75$

Diâmetro equatorial (VE)

$\begin{array}{llll}45,75 \pm 0,38 & 1,91 & 4,18 & 44,97-46,53 \\ 46,62 \pm 0,49 & 2,47 & 5,30 & 45,61-47,63 \\ 50,09 \pm 0,48 & 2,42 & 4,83 & 49,10-51,08 \\ 45,08 \pm 0,40 & 2,00 & 4,44 & 44,26-45,90 \\ 51,07 \pm 0,49 & 2,44 & 4,78 & 50,06-52,08 \\ 59,97 \pm 0,54 & 2,68 & 4,46 & 58,86-61,08 \\ 78,78 \pm 1,83 & 7,34 & 9,32 & 75,01-82,55 \\ 88,97 \pm 1,00 & 5,00 & 5,62 & 86,91-91,03 \\ 37,06 \pm 0,42 & 2,10 & 5,66 & 36,19-37,93 \\ 38,07 \pm 0,46 & 2,30 & 6,04 & 37,12-39,02 \\ 48,91 \pm 0,44 & 2,22 & 4,53 & 48,00-49,82 \\ 44,47 \pm 0,34 & 1,72 & 3,86 & 43,77-45,17 \\ 84,42 \pm 0,49 & 2,43 & 2,88 & 83,41-85,43 \\ 82,00 \pm 0,60 & 2,99 & 3,64 & 80,76-83,24 \\ 78,65 \pm 0,72 & 3,59 & 4,56 & 77,17-80,13 \\ 73,60 \pm 0,78 & 3,89 & 5,29 & 71,99-75,21 \\ 40,90 \pm 0,57 & 2,84 & 6,94 & 39,73-42,07 \\ 40,07 \pm 0,42 & 2,03 & 5,06 & 39,20-40,94\end{array}$

Diâmetro equatorial (VP)

\begin{tabular}{llll}
$44,85 \pm 0,37$ & 1,84 & 4,11 & $44,09-45,61$ \\
$45,57 \pm 0,45$ & 2,23 & 4,89 & $44,64-46,50$ \\
$47,92 \pm 0,48$ & 2,40 & 5,01 & $46,93-48,91$ \\
$43,50 \pm 0,41$ & 2,07 & 4,76 & $42,66-44,34$ \\
$48,01 \pm 0,50$ & 2,50 & 5,20 & $46,98-49,04$ \\
$54,15 \pm 0,57$ & 2,83 & 5,23 & $52,98-55,32$ \\
$79,72 \pm 0,94$ & 4,69 & 5,88 & $77,78-81,66$ \\
$88,06 \pm 1,59$ & 6,35 & 7,21 & $84,78-91,34$ \\
$36,74 \pm 0,41$ & 2,05 & 5,58 & $35,90-37,58$ \\
$36,21 \pm 0,27$ & 1,30 & 3,59 & $35,65-36,77$ \\
$46,63 \pm 0,34$ & 1,70 & 3,65 & $45,93-47,33$ \\
$43,87 \pm 0,31$ & 1,55 & 3,52 & $43,23-44,51$ \\
$80,54 \pm 0,58$ & 2,90 & 3,60 & $79,35-81,73$ \\
$81,20 \pm 0,80$ & 4,02 & 4,95 & $79,55-82,85$ \\
$75,71 \pm 1,28$ & 6,39 & 8,45 & $73,07-78,35$ \\
$72,38 \pm 0,88$ & 4,40 & 6,08 & $70,57-74,19$ \\
$44,15 \pm 0,51$ & 2,54 & 5,75 & $43,10-45,20$ \\
$39,67 \pm 0,43$ & 2,16 & 5,44 & $38,78-40,56$ \\
\hline
\end{tabular}



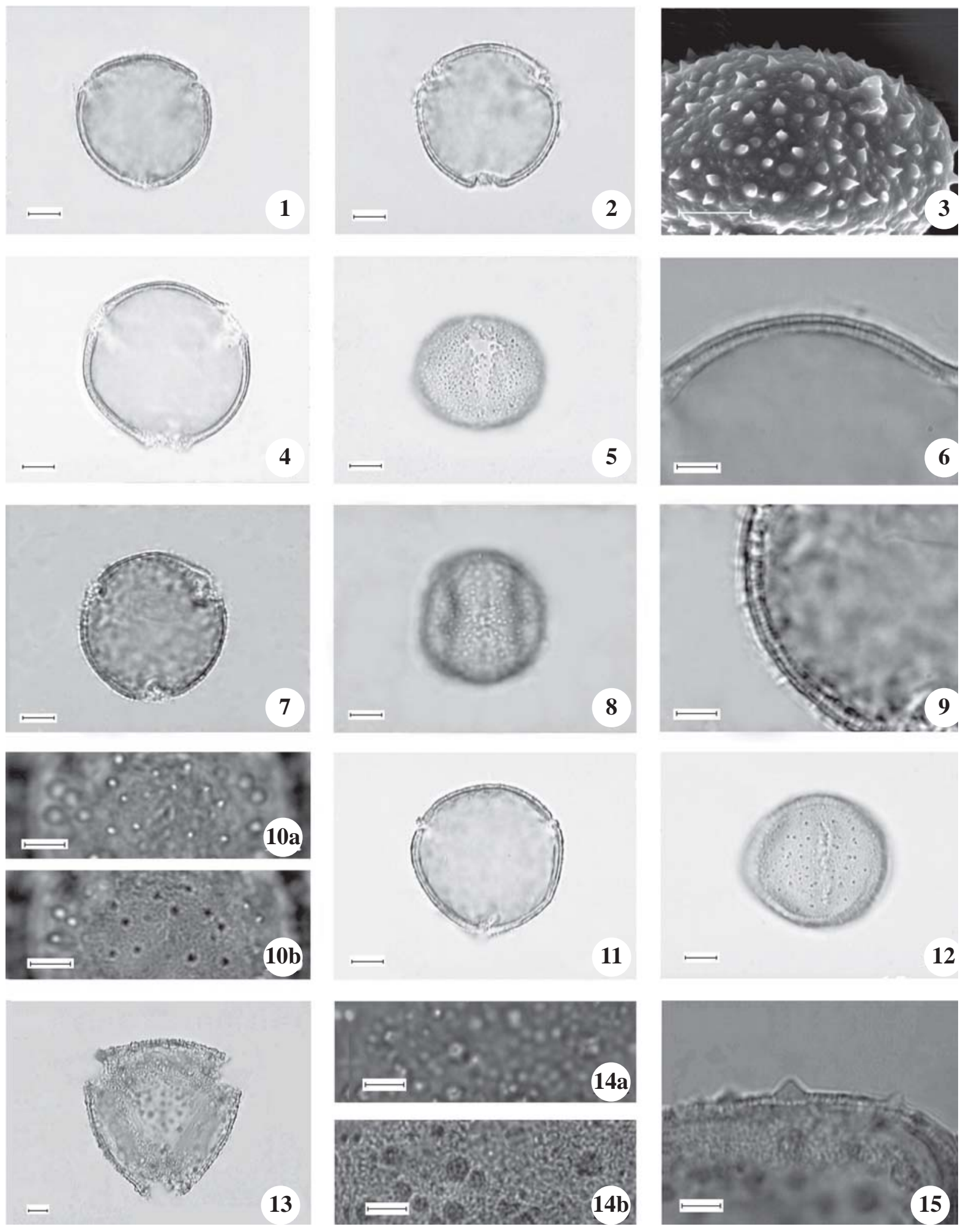

Figuras 1-15. Fotomicrografias e eletromicrografias dos grãos de pólen 3-colpados de espécies de Cordiaceae. 1. Cordia ecalyculata brevistila, Vista polar. 2-3. C. ecalyculata longistila. 2. Vista polar. 3. Detalhe da ornamentação (MEV). 4-6. C. glabrata brevistila. 4. Vista polar. 5. Vista equatorial. 6. Corte óptico. 7-10. C. glabrata longistila. 7. Vista polar. 8. Vista equatorial. 9. Corte óptico. 10. L.O. em foco alto e foco baixo. 11-12. C. magnolifolia brevistila. 11. Vista polar. 12. Vista equatorial. 13-14. C. rufescens brevistila. 13. Vista polar. 14. L.O. em dois níveis de focalização. 15. C. rufescens longistila, Corte óptico. Barra $=5 \mu \mathrm{m}(3,6,9-10,14-15) ; 10 \mu \mathrm{m}(1-2,4-5,7-8,11-13)$. 
raramente subcirculares (C. ecalyculata longistila e $C$. magnoliifolia brevistila, tabela 3), algumas vezes com extremidades afiladas (figura 29), raramente constritas no centro ( $C$. trichotoma brevistila), em alguns casos são de difícil visualização (figura 8) e mensuração. A membrana do cólporo é ornamentada com espículos e grânulos (figura 27). Os grãos de pólen de C. magnoliifolia longistila apresentam colpos e endoaberturas maiores que os das outras espécies estudadas (tabela 3 ).

Em geral teto delgado, camada columelar mais espessa (exceção em C. glabrata longistila, tabela 3 ). Nexina mais espessa que a sexina, exceto em $C$. rufescens, C. sellowiana brevistila, C. superba e $C$. taguahyensis. Observou-se, em ambas as formas florais de $C$. trichotoma, 93\% de grãos de pólen 3-colporados e 7\% de 4-colporados.

Sob MEV observam-se perfurações e grânulos entre os espinhos (figura 28), exceto em $C$. superba que apresenta espículos supra tectais sobre as verrugas.

Morfologicamente os grãos de pólen das flores brevistilas e longistilas neste tipo polínico são similares. Verifica-se, no entanto, que os grãos de pólen das flores longistilas de $C$. ecalyculata, $C$. magnoliifolia, $C$. rufescens e $C$. superba apresentam diâmetro e abertura maiores quando comparados com os grãos de pólen das flores brevistilas. Já os grãos de pólen das flores brevistilas de $C$. glabrata, $C$. silvestris, $C$. trichotoma e $C$. taguahyensis são maiores que os das longistilas, enquanto que em $C$. sellowiana não ocorrem diferenças significativas entre os dois tipos de flores (tabela 2, figura 61). Os grãos de pólen das flores longistilas mostram espinhos maiores e mais altos quando comparados com os das flores brevistilas (exceto em $C$. superba) conferindo uma ornamentação mais nítida e consistente; o mesmo ocorrendo em relação à espessura das camadas da exina (exceto em $C$. silvestris e $C$. trichotoma, tabela 3 ).

Os materiais de comparação analisados apresentaram valores de diâmetros dentro do intervalo de confiança ou da faixa de variação quando comparados com os dos respectivos materiais padrão, exceto nos espécimes Britez 24695, Ivanauskas 676, Leitão Filho et al. 32576, Medri et al. 501, Montilha et al. s.n.
(SJRP19921), Passos \& Kim 37, Sobral 3688 e Souza et al. 32 (tabela 4).

A análise de componentes principais (PCA) foi realizada com 10 variáveis métricas (tabela 1 e 5, figura 62), resumindo em seus dois primeiros eixos $92,8 \%$ da variabilidade total dos dados. No lado esquerdo do eixo 1 foram ordenadas as espécies $C$. rufescens, $C$. superba, $C$ taguahyensis e $C$. magnoliifolia longistila, associadas aos altos valores das variáveis métricas (>0,5 - tabela 5). Diferentemente, as demais espécies podem ser observadas no lado direito do eixo 1 por apresentarem os menores valores métricos.

Tipo II - Grãos de pólen 3-porados (Varronia calocephala, $V$. curassavica, V. discolor, V. guazumaefolia, V. leucocephala, V. monosperma, V. sessilifolia, V. truncata e V. urticifolia) - Grãos de pólen médios a grandes (tabela 6); isopolares; âmbito circular a subcircular; oblatoesferoidais a suboblatos (V. monosperma longistila, tabela 7); 3-porados, poros operculados, recobertos por sexina esculturada, dificultando a visualização destes (figura 34); poros geralmente maiores no comprimento (tabela 7). Sexina semitectada, reticulada, heterorreticulada a homorreticulada ( $V$. calocephala); simplescolumelada, lumens lisos, menores em direção às aberturas. Sob MEV é possível observar com nitidez a presença de espículos sobre os muros (figura 36). Nexina mais delgada que a sexina e subdividida em nexina 1 e nexina 2 (tabela 7 ). Os retículos variam quanto ao tamanho e a forma. Observam-se retículos maiores nos grãos de pólen de $V$. curassavica longistila, $V$. discolor longistila, $V$. leucocephala brevistila, $V$. sessilifolia longistila e $V$. urticifolia brevistila (tabela 7).

Somente os grãos de pólen das flores brevistilas de $V$. monosperma, $V$. sessilifolia e $V$. truncata apresentam diâmetros maiores que os das flores longistilas; nas demais espécies os grãos de pólen das flores longistilas apresentam os maiores diâmetros (tabela 6, figura 63). A ornamentação da exina é mais evidente nos grãos de pólen de flores longistilas, pois possuem lumens maiores que os das brevistilas (exceto em $V$. leucocephala, $V$. monosperma e $V$. urticifolia); $V$. truncata apresenta ornamentação semelhante nos grãos de pólen de ambos tipos de flores.

Figures 1-15. Light and scanning electron micrographs of 3-colpate pollen grains of Cordiaceae. 1. Cordia ecalyculata shortstyle, Polar view. 2-3. C. ecalyculata longstyle. 2. Polar view. 3. Detail of ornamentation (SEM). 4-6. C. glabrata shortstyle. 4. Polar view. 5. Equatorial view. 6. Optical section. 7-10. C. glabrata longstyle. 7. Polar view. 8. Equatorial view. 9. Optical section. 10. L.O. in high and low focus. 11-12. C. magnolifolia shortstyle. 11. Polar view. 12. Equatorial view. 13-14. C. rufescens shortstyle. 13. Polar view. 14. L.O. in high and low focus. 15. C. rufescens longstyle, Optical section. Bar $=5 \mu \mathrm{m}(3,6,9-10,14-15) ; 10 \mu \mathrm{m}(1-2,4-5,7-8,11-13)$. 

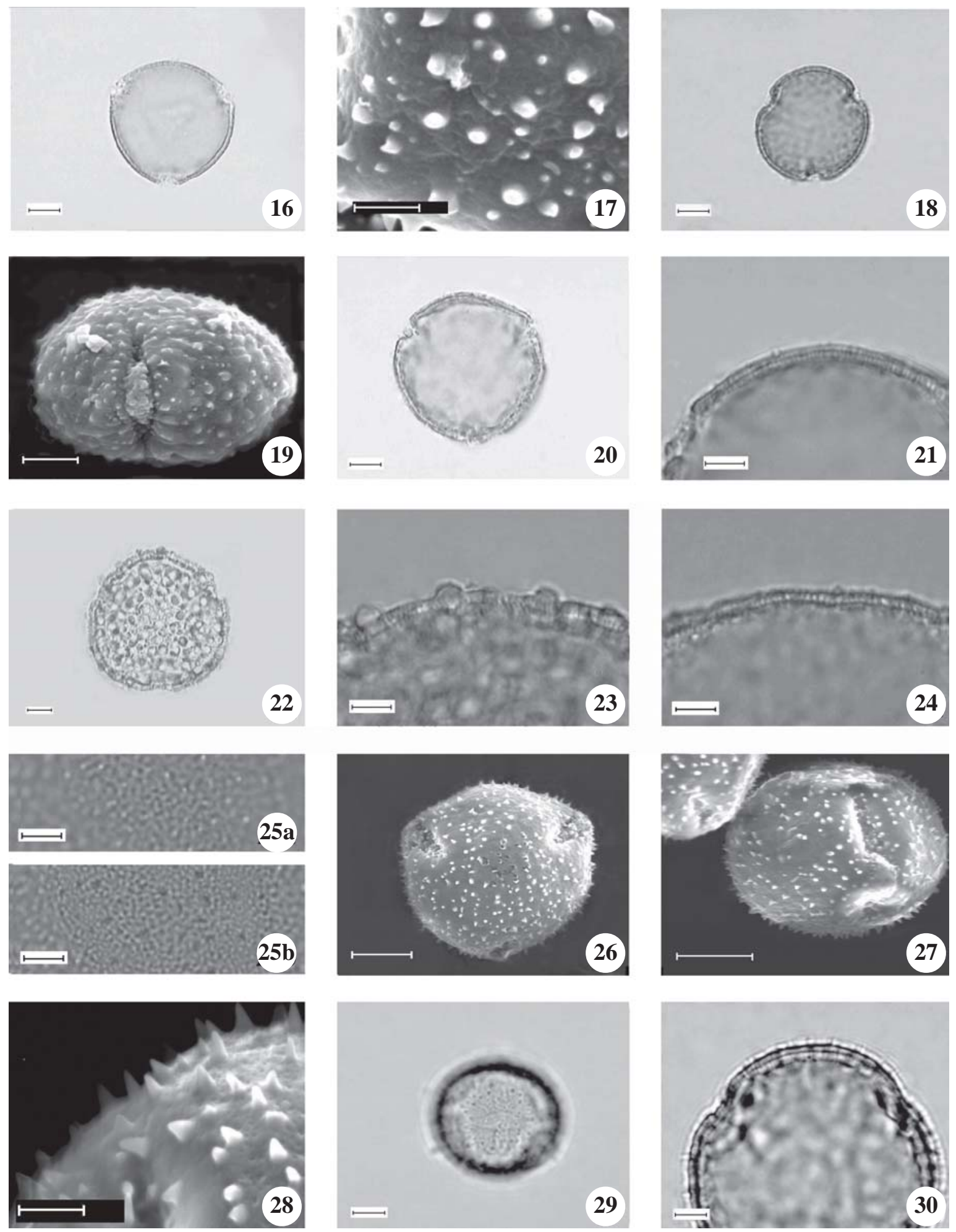

Figuras 16-30. Fotomicrografias e eletromicrografias dos grãos de pólen 3-colpados de espécies de Cordiaceae. 16-17. Cordia sellowiana brevistila. 16. Vista polar. 17. Vista polar, detalhe da ornamentação (MEV). 18-19. C. sellowiana longistila. 18. Vista polar. 19. Vista equatorial evidenciando a abertura (MEV). 20. C. silvestris brevistila, Vista polar. 21. C. silvestris longistila, Corte óptico. 22-23. C. superba brevistila. 22. Vista polar. 23. Corte óptico. 24-25. C. taguahyensis longistila. 24. Corte óptico. 25. L.O. em foco alto e foco baixo. 26-28. C. trichotoma brevistila. 26. Vista polar (MEV). 27. Vista equatorial (MEV). 28. Detalhe da ornamentação (MEV). 29-30. C. trichotoma longistila. 29. Vista equatorial. 30. Corte óptico. Barra $=2 \mu \mathrm{m}(17,22,28) ; 5 \mu \mathrm{m}(19,21,24-25,30) ; 10 \mu \mathrm{m}(16,18,20,23,26-27,29)$. 
Tabela 3. Média aritmética das medidas $(\mu \mathrm{m})$ do colpo, endoabertura e camadas da exina dos grãos de pólen 3-colporados das espécies de Cordiaceae $(n=10)$. $(\mathrm{B}=$ brevistila; $\mathrm{L}=$ longistila; Compr. $=$ comprimento; Larg. $=$ largura; Marg. = margem; Esp. = espinhos; Alt. = altura; Ver. = verrugas; $\mathrm{P}=$ diâmetro polar; $\mathrm{E}=$ diâmetro equatorial).

Table 3. Arithmetic average of measures $(\mu \mathrm{m})$ of colpus, endoaperture exine layers in 3-colporate pollen grains of Cordiaceae $(n=10) .(\mathrm{B}=$ shortstyle; $\mathrm{L}=$ longstyle; Compr. $=$ length; Larg. $=$ width; Marg. $=$ margo; Esp. $=$ spines; Alt. $=$ height; Ver. $=$ verruca; $\mathrm{P}=$ polar diameter; $\mathrm{E}=$ equatorial diameter).

\begin{tabular}{|c|c|c|c|c|c|c|c|c|c|c|c|c|}
\hline \multirow{2}{*}{ Espécies de Cordia } & \multicolumn{3}{|c|}{ Colpo } & \multicolumn{2}{|c|}{ Endoabertura } & \multirow{2}{*}{ Exina } & \multirow{2}{*}{ Sexina } & \multirow{2}{*}{ Nexina } & \multirow{2}{*}{ Teto } & \multirow{2}{*}{$\frac{\text { Esp. }}{\text { Alt. }}$} & \multirow{2}{*}{$\frac{\text { Ver. }}{\text { Alt. }}$} & \multirow[b]{2}{*}{$\mathrm{P} / \mathrm{E}$} \\
\hline & Compr. & Larg. & Marg. & Compr. & Larg. & & & & & & & \\
\hline C. ecalyculata (B) & 21,95 & 2,49 & - & 4,34 & 5,08 & 4,56 & 1,21 & 1,49 & 0,59 & 1,86 & - & 0,87 \\
\hline C. ecalyculata (L) & 26,80 & 2,71 & - & 5,39 & 5,61 & 4,66 & 1,35 & 1,59 & 0,64 & 1,72 & - & 0,87 \\
\hline C. glabrata (B) & 31,61 & 2,41 & 2,85 & 6,34 & 13,14 & 3,91 & 1,06 & 1,55 & 0,53 & 1,30 & - & 0,86 \\
\hline C. glabrata $(\mathrm{L})$ & 28,84 & 2,98 & 2,96 & 5,32 & 9,20 & 4,38 & 1,13 & 1,64 & 0,62 & 1,61 & - & 0,96 \\
\hline C. magnoliifolia (B) & 30,86 & 3,03 & - & 5,10 & 5,16 & 4,64 & 1,31 & 1,66 & 0,68 & 1,67 & - & 0,87 \\
\hline C. magnoliifolia (L) & 35,13 & 5,34 & - & $11,63^{*}$ & $12,71^{*}$ & 4,94 & 1,40 & 1,76 & 0,75 & 1,78 & - & 0,85 \\
\hline C. rufescens (B) & $53,25^{*}$ & $14,88^{*}$ & - & $9,75^{*}$ & $15,50 *$ & 6,75 & 2,36 & 1,66 & 0,96 & 2,73 & - & 0,88 \\
\hline C. rufescens $(\mathrm{L})$ & $51,88^{*}$ & $8,13^{*}$ & - & $9,38 *$ & $13,06^{*}$ & 7,27 & 2,22 & 1,56 & 1,04 & 3,49 & - & 0,88 \\
\hline C. sellowiana $(\mathrm{B})$ & 21,58 & 2,69 & - & 5,26 & 8,03 & 2,87 & 0,87 & 0,86 & 0,49 & 1,14 & - & 0,87 \\
\hline C. sellowiana $(\mathrm{L})$ & 22,40 & 3,94 & - & 5,25 & 8,81 & 3,52 & 1,07 & 1,12 & 0,63 & 1,33 & - & 0,85 \\
\hline C. silvestris (B) & 30,95 & 3,58 & - & 6,18 & 8,91 & 4,89 & 1,33 & 1,80 & 0,71 & 1,76 & - & 0,90 \\
\hline C. silvestris (L) & 18,04 & 3,75 & - & 5,00 & 7,84 & 4,32 & 1,24 & 1,45 & 0,72 & 1,63 & - & 0,89 \\
\hline C. superba (B) & $59,13^{*}$ & $12,00 *$ & - & - & - & 6,23 & 1,84 & 1,81 & 0,80 & - & 2,58 & 0,86 \\
\hline C. superba (L) & $49,50 *$ & $4,50 *$ & - & - & - & 6,06 & 2,02 & 1,54 & 0,87 & - & 2,50 & 0,87 \\
\hline C. taguahyensis (B) & $40,57 *$ & $4,63^{*}$ & - & $8,75^{*}$ & $9,25^{*}$ & 5,29 & 2,06 & 1,70 & 0,92 & 1,53 & - & 0,91 \\
\hline C. taguahyensis $(\mathrm{L})$ & $45,00 *$ & $6,71^{*}$ & - & $6,13^{*}$ & $7,75^{*}$ & 5,20 & 1,98 & 1,57 & 0,89 & 1,65 & - & 0,91 \\
\hline C. trichotoma (B) & 24,83 & 3,12 & 4,43 & 6,50 & 10,40 & 3,53 & 1,05 & 1,45 & 0,45 & 1,03 & - & 0,96 \\
\hline C. trichotoma $(\mathrm{L})$ & 22,09 & 3,13 & 4,47 & 4,50 & 11,89 & 4,03 & 1,14 & 1,44 & 0,49 & 1,45 & - & 0,94 \\
\hline
\end{tabular}

$* n<10$.

Dentre os materiais de comparação analisados, somente os espécimes Assis et al. 300, Hoffmann et al. 62, 66, Leitão Filho et al. 34652, Souza 9472, Souza et al. 3584, 11203 e Taroda s.n. (SJRP20333) apresentaram valores de diâmetros dentro do intervalo de confiança ou da faixa de variação quando comparados com os dos respectivos materiais padrão (tabela 8 ).

Para a ordenação gráfica das espécies 3-poradas através da análise de componentes principais foram utilizadas nove variáveis métricas dos grãos de pólen (tabela 1, 9). Os dois primeiros eixos representaram
$74,1 \%$ da variância total dos dados (figura 64). O primeiro eixo indicou $61,4 \%$ da variabilidade total, tendo como variáveis mais correlacionadas $(>0,7)$ as medidas dos diâmetros e das aberturas, por outro lado, os valores de espessura do teto $(0,001)$ apresentaram a menor influência na ordenação (tabela 9). O eixo 2 resumiu $12,7 \%$ da variância dos dados tendo como variáveis mais correlacionada a largura e o comprimento do poro (> 0,5, tabela 9).

V. calocephala, V. leucocephala e V. sessilifolia ficaram ordenadas no lado esquerdo do eixo 1 pois

Figures 16-30. Light and scanning electron micrographs of 3-colpate pollen grains of Cordiaceae. 16-17. Cordia sellowiana shortstyle. 16. Polar view. 17. Polar view, detail of ornamentation (SEM). 18-19. C. sellowiana longstyle. 18. Polar view. 19. Equatorial view showing aperture (SEM). 20. C. silvestris shortstyle, Polar view. 21. C. silvestris longstyle, Optical section. 22-23. C. superba shortstyle. 22. Polar view. 23. Optical section. 24-25. C. taguahyensis longstyle. 24. Optical section. 25. L.O. in high focus. 26-28. C. trichotoma shortstyle. 26. Polar view (SEM). 27. Equatorial view (SEM). 28. Detail of ornamentation (SEM). 29-30. C. trichotoma longstyle. 29. Equatorial view. 30. Optical section. Bar $=2 \mu \mathrm{m}(17,22,28) ; 5 \mu \mathrm{m}$ $(19,21,24-25,30) ; 10 \mu \mathrm{m}(16,18,20,23,26-27,29)$. 

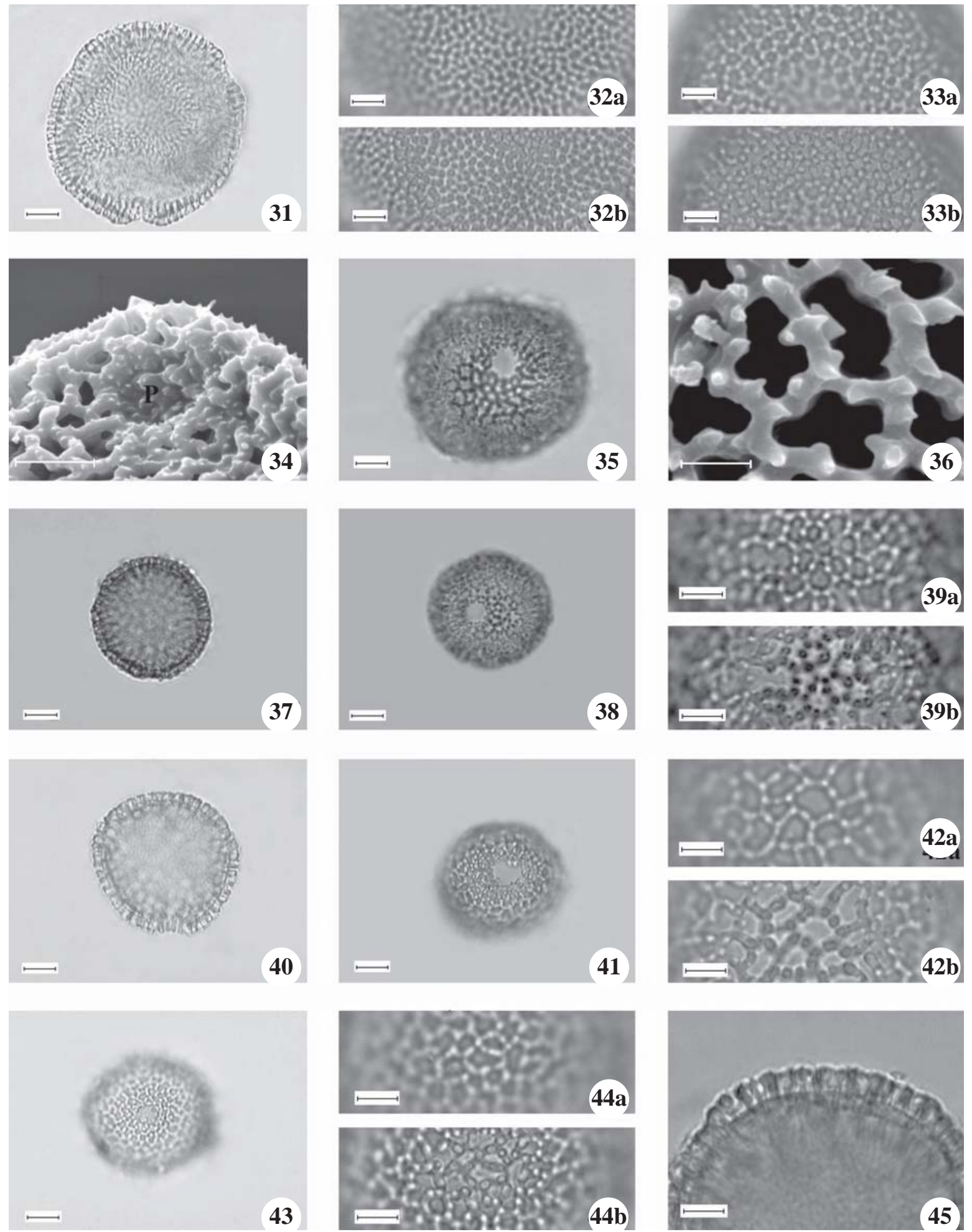

Figuras 31-45. Fotomicrografias e eletromicrografias dos grãos de pólen 3-porados de espécies de Cordiaceae. 31-32. Varronia calocephala brevistila. 31. Vista polar. 32. L.O. em foco alto e foco baixo. 33. V. calocephala longistila, L.O. em foco alto e foco baixo. 34. V. curassavica brevistila, Vista equatorial evidenciando a abertura (MEV). 35-36. V. curassavica longistila. 35. Vista equatorial. 36. Detalhe da ornamentação (MEV). 37-39. V. discolor brevistila. 37. Vista polar. 38. Vista equatorial. 39. L.O. em foco alto e foco baixo. 40-42. V. discolor longistila. 40. Vista polar. 41. Vista equatorial. 42. L.O. em foco alto e foco baixo. 43-44. V. guazumaefolia brevistila. 43. Vista equatorial. 44. L.O. em foco alto e foco baixo. 45. V. guazumaefolia longistila, Corte óptico. Barra = $2 \mu \mathrm{m}(36) ; 5 \mu \mathrm{m}(32-34,39,42,44-45) ; 10 \mu \mathrm{m}(31,35,37-38,40-41,43)$. 
Tabela 4. Média aritmética das medidas $(\mu \mathrm{m})$ dos grãos de pólen 3-colporados dos materiais de comparação de Cordiaceae $(n=10)$. ( $\mathrm{B}=$ brevistila; $\mathrm{L}=$ longistila; $\mathrm{P}=$ diâmetro polar; $\mathrm{E}=$ diâmetro equatorial $)$.

Table 4. Measure $(\mu \mathrm{m})$ of arithmetic average of 3-colporate pollen grains obtained from species of comparison of Cordiaceae $(n=10)$. ( $\mathrm{B}=$ shortstyle; $\mathrm{L}=$ longstyle; $\mathrm{P}=$ polar diameter; $\mathrm{E}=$ equatorial diameter $)$.

\begin{tabular}{|c|c|c|c|c|}
\hline \multirow[b]{2}{*}{ Espécies de Cordia/coletores } & \multicolumn{2}{|c|}{ Vista equatorial } & \multirow{2}{*}{$\begin{array}{c}\text { Vista polar } \\
\text { Diâmetro } \\
\text { equatorial }\end{array}$} & \multirow[b]{2}{*}{$\mathrm{P} / \mathrm{E}$} \\
\hline & $\begin{array}{c}\text { Diâmetro } \\
\text { polar }\end{array}$ & $\begin{array}{l}\text { Diâmetro } \\
\text { equatorial }\end{array}$ & & \\
\hline \multicolumn{5}{|l|}{ C. ecalyculata (B) } \\
\hline Assis et al. 1236 & 43,01 & 48,08 & 47,68 & 0,89 \\
\hline Gehrt s.n. (SP5304) & 42,80 & 47,34 & 46,71 & 0,90 \\
\hline Medri et al. 501 & 49,75 & 55,35 & 50,96 & 0,90 \\
\hline Sobral 3688 & 47,04 & 52,44 & 49,81 & 0,90 \\
\hline \multicolumn{5}{|l|}{ C. ecalyculata (L) } \\
\hline Furlan 207 & 42,73 & 49,13 & 46,59 & 0,87 \\
\hline Gibbs \& Leitão Filho 6093 & 43,21 & 47,78 & 45,16 & 0,91 \\
\hline Kinupp \& Francisco 946 & 37,44 & 45,26 & 42,60 & 0,83 \\
\hline \multicolumn{5}{|l|}{ C. glabrata $(\mathrm{B})$} \\
\hline E.P.Heringer \& Paula 13922 & 42,55 & 46,63 & 46,40 & 0,91 \\
\hline Trigo 15121 & 45,51 & 48,21 & 48,80 & 0,94 \\
\hline \multicolumn{5}{|l|}{ C. glabrata $(\mathrm{L})$} \\
\hline Borges 35469 & 43,69 & 44,99 & 47,70 & 0,97 \\
\hline Hatschbach 40134 & 39,43 & 44,54 & 44,75 & 0,89 \\
\hline Lorenzi s.n. (SP262131) & 41,86 & 48,10 & 47,05 & 0,87 \\
\hline Souza 8354 & 38,75 & 43,20 & 43,49 & 0,90 \\
\hline \multicolumn{5}{|l|}{ C. magnoliifolia (B) } \\
\hline Hoehne s.n. (SPF11629) & 50,14 & 53,89 & 53,23 & 0,93 \\
\hline \multicolumn{5}{|l|}{ C. rufescens $(\mathrm{L})$} \\
\hline Vieira et al. 240 & 82,78 & 91,58 & 86,23 & 0,90 \\
\hline \multicolumn{5}{|l|}{ C. sellowiana $(\mathrm{B})$} \\
\hline Britez 24695 & 39,00 & 42,45 & 42,11 & 0,92 \\
\hline Cielo Filho 184 & 35,05 & 40,25 & 36,73 & 0,87 \\
\hline Ivanauskas 148 & 38,79 & 40,41 & 38,29 & 0,96 \\
\hline Ivanauskas 676 & 37,25 & 44,38 & 41,71 & 0,84 \\
\hline Meira Neto et al. 21515 & 33,65 & 37,70 & 36,22 & 0,89 \\
\hline \multicolumn{5}{|l|}{ C. silvestris $(\mathrm{B})$} \\
\hline Assis 44 & 43,43 & 49,66 & 45,62 & 0,87 \\
\hline Silva 10995 & 38,00 & 41,74 & 40,97 & 0,91 \\
\hline \multicolumn{5}{|l|}{ C. superba (B) } \\
\hline Marinis 545 & 70,05 & 79,60 & 78,60 & 0,88 \\
\hline
\end{tabular}

Figures 31-45. Light and scanning electron micrographs of 3-porate pollen grains of Cordiaceae. 31-32. V. calocephala shortstyle. 31. Polar view. 32. L.O. in high and low focus. 33. V. calocephala longstyle, L.O. in high and low focus. $34 . V$. curassavica shortstyle, Equatorial view showing aperture (SEM). 35-36. V. curassavica longstyle. 35. Equatorial view. 36. Detail of ornamentation (SEM). 37-39. V. discolor shortstyle. 37. Polar view. 38. Equatorial view. 39. L.O. in high and low focus. 40-42. V. discolor longstyle. 40. Polar view. 41. Equatorial view. 42. L.O. in high and low focus. 43-44. V. guazumaefolia shortstyle. 43. Equatorial view. 44. L.O. in high and low focus. 45. V. guazumaefolia longstyle, Optical section. Bar $=2 \mu \mathrm{m}$ (36); $5 \mu \mathrm{m}(32-34,39,42,44-45) ; 10 \mu \mathrm{m}(31,35,37-38,40-41,43)$. 
continuação

\begin{tabular}{|c|c|c|c|c|}
\hline \multirow[b]{2}{*}{ Espécies de Cordia/coletores } & \multicolumn{2}{|c|}{ Vista equatorial } & \multirow{2}{*}{$\begin{array}{c}\text { Vista polar } \\
\text { Diâmetro } \\
\text { equatorial }\end{array}$} & \multirow[b]{2}{*}{$\mathrm{P} / \mathrm{E}$} \\
\hline & $\begin{array}{l}\text { Diâmetro } \\
\text { polar }\end{array}$ & $\begin{array}{l}\text { Diâmetro } \\
\text { equatorial }\end{array}$ & & \\
\hline \multicolumn{5}{|l|}{ C. superba $(\mathrm{L})$} \\
\hline Cavalcanti 261 & 83,33 & 91,56 & 84,64 & 0,91 \\
\hline César et al. s $n$. (HRCB11286) & 73,23 & 84,59 & 77,14 & 0,87 \\
\hline Godoi et al. 283 & 69,09 & 82,44 & 74,64 & 0,84 \\
\hline Furlan 201 & 75,86 & 84,36 & 78,95 & 0,90 \\
\hline Tamashiro et al. 685 & 79,44 & 89,04 & 84,13 & 0,89 \\
\hline \multicolumn{5}{|l|}{ C. taguahyensis (L) } \\
\hline Gehrt s n. (SP44380) & 63,61 & 73,91 & 70,16 & 0,86 \\
\hline Leitão Filho et al. 32576 & 80,59 & 89,88 & 85,29 & 0,90 \\
\hline \multicolumn{5}{|l|}{ C. trichotoma $(\mathrm{B})$} \\
\hline Bernaci et al. 1638 & 41,48 & 45,18 & 42,24 & 0,92 \\
\hline Montilha et al. s.n. (SJRP19921) & 38,79 & 43,65 & 39,85 & 0,89 \\
\hline Passos \& Kim 37 & 45,29 & 48,18 & 47,44 & 0,94 \\
\hline Shepherd \& Kock 14 & 44,72 & 45,77 & 43,40 & 0,98 \\
\hline Souza et al. 32 & 35,95 & 40,43 & 38,25 & 0,89 \\
\hline Traldi \& Souza 25 & 39,61 & 44,10 & 43,05 & 0,90 \\
\hline \multicolumn{5}{|l|}{ C. trichotoma $(\mathrm{L})$} \\
\hline Gibbs 4781 & 39,29 & 43,16 & 42,97 & 0,91 \\
\hline Santin 30925 & 36,28 & 42,33 & 40,52 & 0,86 \\
\hline
\end{tabular}

Tabela 5. Coeficientes de correlação de Pearson e Kendall entre as variáveis métricas dos grãos de pólen 3-colpados e os dois primeiros eixos da ordenação pela PCA para as espécies estudadas de Cordiaceae.

Table 5. Pearson and Kendall correlation coefficients of metric variables between 3-colpate pollen grains and the first two axis of PCA ordination, family Cordiaceae.

\begin{tabular}{llcc}
\hline \multirow{2}{*}{ Variáveis } & \multicolumn{2}{c}{ Componentes Principais } \\
\cline { 3 - 4 } DPVE & (diâmetro polar em vista equatorial) & $-0,963$ & Eixo 2 \\
DEVE & (diâmetro equatorial em vista equatorial) & $-0,911$ & $-0,217$ \\
DEVP & (diâmetro equatorial em vista polar) & $-0,969$ & $-0,254$ \\
CCOM & (comprimento do colpo) & $-0,952$ & $-0,184$ \\
CLAR & (largura do colpo) & $-0,898$ & $-0,123$ \\
EXIN & (espessura da exina) & $-0,905$ & $+0,439$ \\
SEXI & (espessura da sexina) & $-0,949$ & $-0,206$ \\
NEXI & (espessura da nexina) & $-0,552$ & $-0,093$ \\
TETO & (espessura do teto) & $-0,886$ & $-0,322$ \\
ESPI & (altura dos espinhos) & $-0,836$ & $-0,069$ \\
\end{tabular}

apresentaram os maiores valores para todas as variáveis métricas analisadas em seus grãos de pólen, as demais espécies estão localizadas no lado direito deste primeiro eixo evidenciando as menores medidas observadas; posição intermediária foi ocupada pelas formas longistilas de $V$. curassavica e V. urticifolia (figura 64). No primeiro eixo é interessante notar ainda, a proximidade entre as formas brevistilas e longistilas para os grãos de pólen de $V$. discolor (lado direito) e V. sessilifolia (lado esquerdo). As espécies V. sessilifolia brevistila e longistila, 

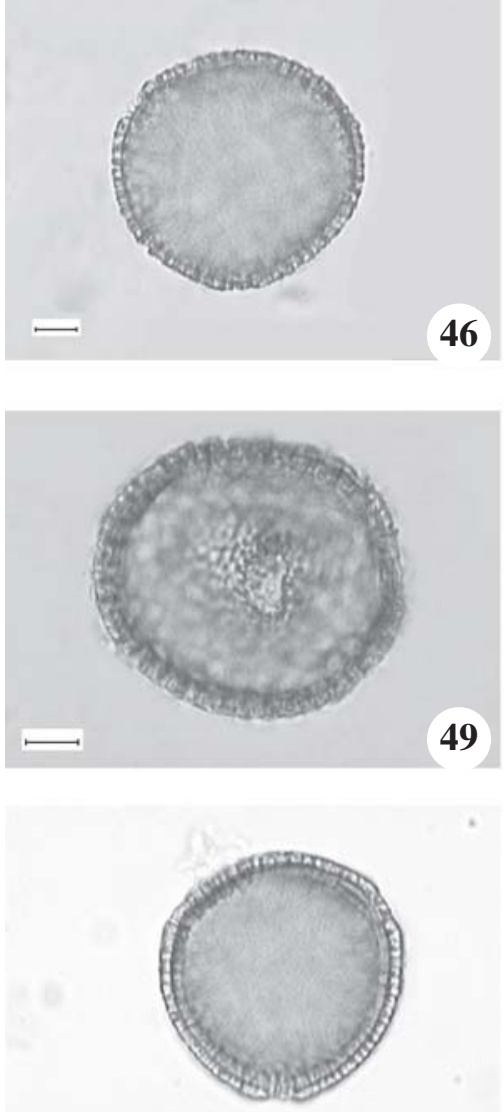

52
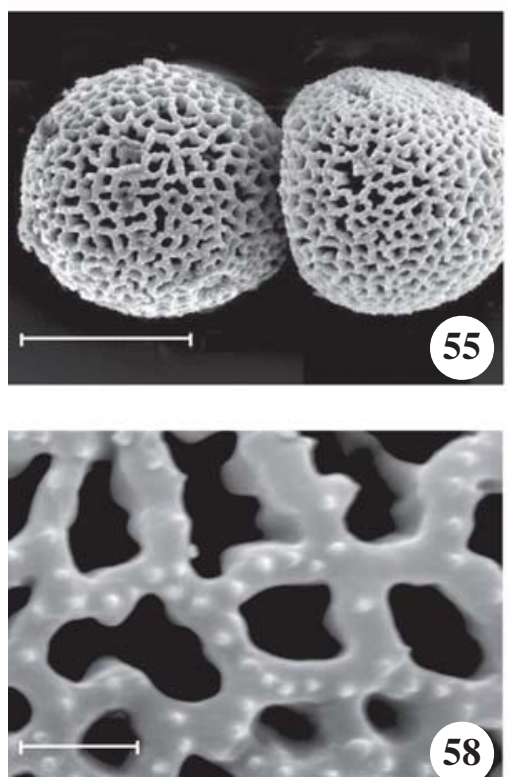

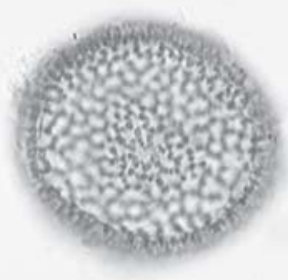

47
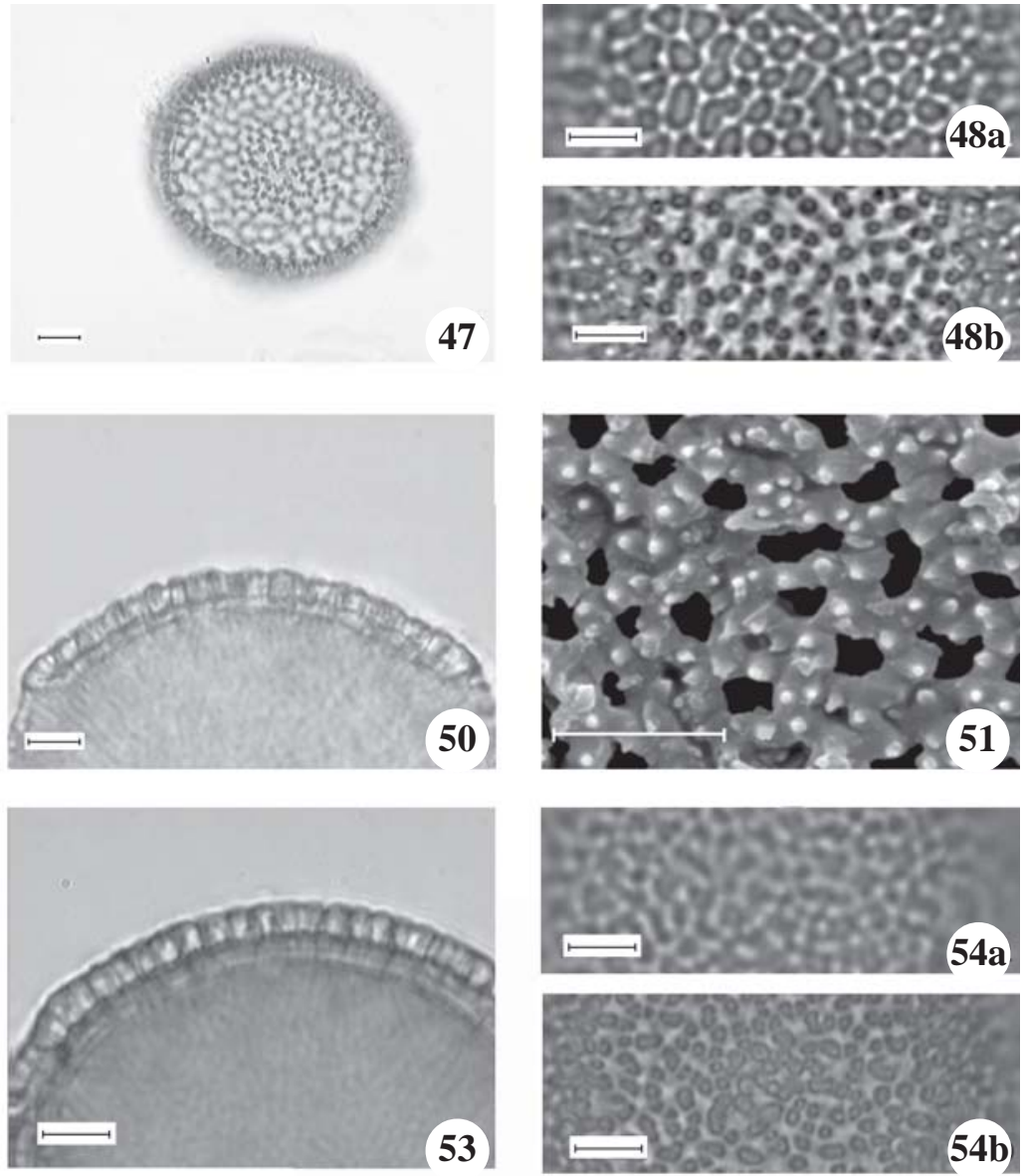

53
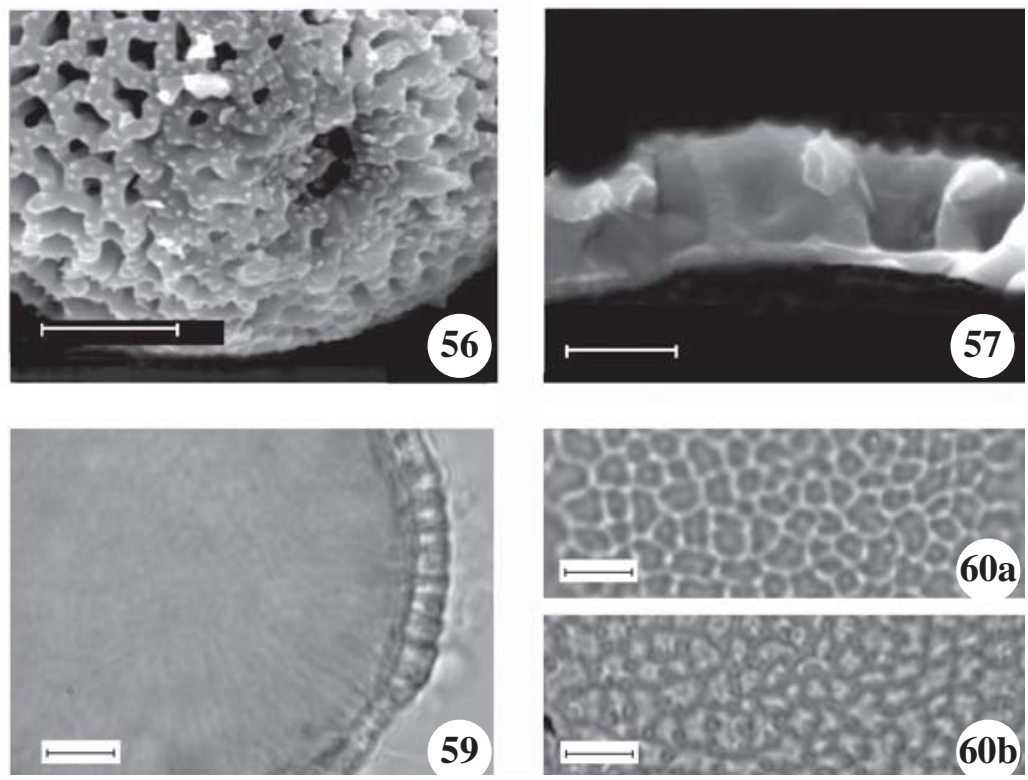
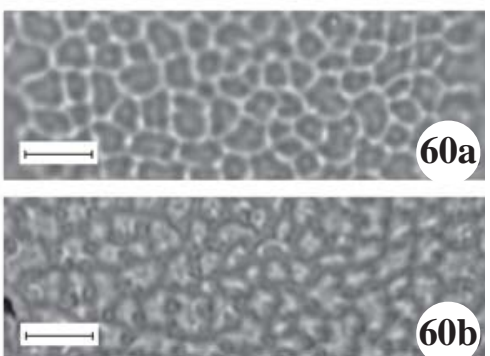

Figuras 46-60. Fotomicrografias e eletromicrografias dos grãos de pólen 3-porados de espécies de Cordiaceae. 46-47. Varronia leucocephala brevistila. 46. Vista polar. 47. Equatorial view. 48. V. monosperma brevistila, L.O. em foco alto e foco baixo. 49. V. sessilifolia brevistila, Vista equatorial. 50-51. V. sessilifolia longistila. 50. Corte óptico. 51. Detalhe da ornamentação (MEV). 52-53. V. truncata brevistila. 52. Vista polar. 53. Corte óptico. 54. V. truncata longistila, L.O. em foco alto e foco baixo. 55-58. V. urticifolia brevistila. 55. Vista geral de dois grãos de pólen (MEV). 56. Detalhe da abertura (MEV). 57. Detalhe da exina (MEV). 58. Detalhe da ornamentação (MEV). 59-60. V. urticifolia longistila. 59. Corte óptico. 60. L.O. em foco alto e foco baixo. Barra $=2 \mu \mathrm{m}(57-58) ; 5 \mu \mathrm{m}(48,50-51,53-54,56,59-60) ; 10 \mu \mathrm{m}(46-47,49,52) ; 20 \mu \mathrm{m}(55)$. 
Tabela 6. Medidas dos grãos de pólen 3-porados de espécies de Cordiaceae, em vista equatorial e polar $(n=25)$. (VE $=$ vista equatorial; $\mathrm{VP}=$ vista polar; $\mathrm{B}=$ brevistila; $\mathrm{L}=$ longistila; $x=$ média aritmética; $s_{x}=$ desvio padrão da média; $s=$ desvio padrão da amostra; $\mathrm{V}=$ coeficiente de variabilidade; $\mathrm{IC}=$ intervalo de confiança).

Table 6. Measures of 3-porate pollen grains of Cordiaceae, in equatorial and polar views $(n=25)$. (VE $=$ equatorial view; VP $=$ polar view $; \mathrm{B}=$ shortsyle $\mathrm{L}=$ longstyle $x=$ arithmetic mean; $s_{x}=$ medium standard deviation; $s=$ sample standard deviation; $\mathrm{V}=$ coefficient of variability; I.C. = confidence interval).

\begin{tabular}{|c|c|c|c|c|c|}
\hline Espécies de Varronia & $\begin{array}{l}\text { Faixa de variação } \\
\qquad(\mu \mathrm{m})\end{array}$ & $\begin{array}{l}x \pm s_{x} \\
(\mu \mathrm{m})\end{array}$ & $\begin{array}{c}s \\
(\mu \mathrm{m})\end{array}$ & $\begin{array}{l}\mathrm{V} \\
(\%)\end{array}$ & $\begin{array}{l}\text { I.C. } \\
(\mu \mathrm{m})\end{array}$ \\
\hline & \multicolumn{5}{|c|}{ Diâmetro polar (VE) } \\
\hline V. calocephala (B) & $50,00-58,88$ & $53,83 \pm 0,51$ & 2,57 & 4,77 & $52,77-54,88$ \\
\hline V. calocephala $(\mathrm{L})$ & $52,00-62,38$ & $58,11 \pm 0,49$ & 2,47 & 4,25 & $57,09-59,13$ \\
\hline V. curassavica (B) & $40,00-49,50$ & $45,76 \pm 0,70$ & 2,88 & 6,28 & $44,32-47,20$ \\
\hline V. curassavica (L) & $41,63-55,25$ & $48,66 \pm 0,71$ & 3,53 & 7,25 & $47,20-50,12$ \\
\hline V. discolor (B) & $35,50-41,75$ & $38,44 \pm 0,29$ & 1,44 & 3,74 & $37,84-39,04$ \\
\hline V. discolor $(\mathrm{L})$ & $35,00-43,50$ & $39,24 \pm 0,46$ & 2,29 & 5,83 & $38,29-40,19$ \\
\hline V. guazumaefolia (B) & $32,75-44,25$ & $37,62 \pm 0,54$ & 2,71 & 7,21 & $36,50-38,75$ \\
\hline V. guazumaefolia (L) & $38,13-49,50$ & $41,86 \pm 0,63$ & 3,17 & 7,58 & $40,55-43,16$ \\
\hline V. leucocephala (B) & $57,00-65,75$ & $61,84 \pm 0,43$ & 2,17 & 3,51 & $60,95-62,75$ \\
\hline V. leucocephala (L) & $58,50-74,25$ & $67,47 \pm 0,82$ & 4,12 & 6,11 & $65,77-69,16$ \\
\hline$V$. monosperma $(\mathrm{B})$ & $44,25-52,75$ & $48,56 \pm 0,48$ & 2,39 & 4,92 & $47,57-49,55$ \\
\hline$V$. monosperma $(\mathrm{L})$ & $35,63-50,00$ & $43,49 \pm 0,84$ & 4,21 & 9,69 & $41,75-45,23$ \\
\hline$V$. sessilifolia $(\mathrm{B})$ & $51,38-61,38$ & $56,65 \pm 0,48$ & 2,38 & 4,20 & $55,67-57,63$ \\
\hline V. sessilifolia $(\mathrm{L})$ & $48,63-60,13$ & $55,46 \pm 0,62$ & 3,08 & 5,56 & $54,19-56,72$ \\
\hline V. truncata $(\mathrm{B})$ & $39,50-50,00$ & $44,45 \pm 0,50$ & 2,52 & 5,66 & $43,41-45,49$ \\
\hline V. truncata $(\mathrm{L})$ & $39,38-47,13$ & $44,10 \pm 0,38$ & 1,90 & 4,30 & $43,32-44,88$ \\
\hline V. urticaefolia (B) & $41,25-50,00$ & $45,57 \pm 0,54$ & 2,70 & 5,92 & $44,45-46,68$ \\
\hline \multirow[t]{2}{*}{ V. urticaefolia $(\mathrm{L})$} & $43,63-55,50$ & $50,04 \pm 0,66$ & 3,32 & 6,64 & $48,67-51,40$ \\
\hline & \multicolumn{5}{|c|}{ Diâmetro equatorial (VE) } \\
\hline V. calocephala $(\mathrm{B})$ & $54,25-66,50$ & $59,46 \pm 0,63$ & 3,14 & 5,28 & $58,17-60,75$ \\
\hline V. calocephala $(\mathrm{L})$ & $58,88-64,75$ & $61,36 \pm 0,27$ & 1,35 & 2,20 & $60,80-61,92$ \\
\hline V. curassavica $(\mathrm{B})$ & $45,00-55,25$ & $50,18 \pm 0,70$ & 2,88 & 5,73 & $48,74-51,62$ \\
\hline V. curassavica $(\mathrm{L})$ & $45,63-57,38$ & $52,63 \pm 0,57$ & 2,87 & 5,46 & $51,46-53,80$ \\
\hline V. discolor (B) & $38,50-44,13$ & $41,32 \pm 0,36$ & 1,78 & 4,30 & $40,58-42,06$ \\
\hline V. discolor $(\mathrm{L})$ & $39,00-47,25$ & $41,96 \pm 0,43$ & 2,13 & 5,07 & $41,07-42,85$ \\
\hline V. guazumaefolia (B) & $37,00-48,38$ & $41,55 \pm 0,58$ & 2,91 & 6,99 & $40,35-42,75$ \\
\hline V. guazumaefolia $(\mathrm{L})$ & $40,75-54,50$ & $45,86 \pm 0,58$ & 2,89 & 6,31 & $44,66-47,05$ \\
\hline V. leucocephala (B) & $62,00-75,00$ & $66,56 \pm 0,72$ & 3,58 & 5,38 & $65,08-68,04$ \\
\hline V. leucocephala (L) & $65,63-79,25$ & $73,41 \pm 0,73$ & 3,65 & 4,97 & $71,90-74,91$ \\
\hline$V$. monosperma $(\mathrm{B})$ & $48,13-55,88$ & $51,25 \pm 0,45$ & 2,24 & 4,37 & $50,32-52,18$ \\
\hline$V$. monosperma $(\mathrm{L})$ & $44,88-53,75$ & $49,45 \pm 0,54$ & 2,70 & 5,45 & $48,34-50,56$ \\
\hline$V$. sessilifolia $(\mathrm{B})$ & $60,59-62,27$ & $61,43 \pm 0,41$ & 2,04 & 3,31 & $60,59-62,27$ \\
\hline V. sessilifolia $(\mathrm{L})$ & $55,25-64,63$ & $59,98 \pm 0,53$ & 2,65 & 4,42 & $58,89-61,07$ \\
\hline V. truncata (B) & $46,38-53,63$ & $50,03 \pm 0,38$ & 1,91 & 3,82 & $\begin{array}{r}49,24-50,82 \\
\text { continus }\end{array}$ \\
\hline
\end{tabular}

Figures 46-50. Light and scanning electron micrographs of 3-porate pollen grains of Cordiaceae. 46-47. Varronia leucocephala shortstyle. 46. Polar view. 47. Equatorial view. 48. V. monosperma shortstyle, L.O. in high and low focus. 49. V. sessilifolia shortstyle, Equatorial view. 50-51. V. sessilifolia longstyle. 50. Optical section. 51. Detail of ornamentation (SEM). 52-53. V. truncata shortstyle. 52. Polar view. 53. Optical section. 54. V. truncata longstyle, L.O. in high and low focus. 55-58. V. urticifolia shortstyle. 55. Geral view in two pollen grains (SEM). 56. Detail of aperture (SEM). 57. Detail of exine (SEM). 58. Detail of ornamentation (SEM). 59-60. V. urticifolia longstyle. 59. Optical section. 60. L.O. in high and low focus. Bar $=2 \mu \mathrm{m}$ (57-58); 5 um (48, 50-51, 53-54, 56, 59-60); $10 \mu \mathrm{m}$ (46-47, 49, 52); $20 \mu \mathrm{m}(55)$. 
continuação

\begin{tabular}{|c|c|c|c|c|c|}
\hline Espécies de Varronia & $\begin{array}{l}\text { Faixa de variação } \\
\qquad(\mu \mathrm{m})\end{array}$ & $\begin{array}{c}x \pm s_{x} \\
(\mu \mathrm{m})\end{array}$ & $\begin{array}{c}s \\
(\mu \mathrm{m})\end{array}$ & $\begin{array}{l}\mathrm{V} \\
(\%)\end{array}$ & $\begin{array}{l}\text { I.C. } \\
(\mu \mathrm{m})\end{array}$ \\
\hline V. truncata $(\mathrm{L})$ & $45,25-54,38$ & $49,40 \pm 0,44$ & 2,18 & 4,41 & $48,50-50,30$ \\
\hline V. urticaefolia (B) & $44,00-53,63$ & $48,66 \pm 0,51$ & 2,54 & 5,21 & $47,61-49,71$ \\
\hline \multirow[t]{2}{*}{ V. urticaefolia $(\mathrm{L})$} & $49,50-61,50$ & $55,36 \pm 0,62$ & 3,12 & 5,64 & $54,07-56,64$ \\
\hline & \multicolumn{5}{|c|}{ Diâmetro equatorial (VP) } \\
\hline V. calocephala $(\mathrm{B})$ & $52,38-64,13$ & $56,99 \pm 0,59$ & 2,93 & 5,14 & $55,78-58,20$ \\
\hline V. calocephala $(\mathrm{L})$ & $57,00-65,75$ & $60,99 \pm 0,48$ & 2,42 & 3,97 & $59,99-61,98$ \\
\hline V. curassavica $(\mathrm{B})$ & $40,00-53,00$ & $45,53 \pm 0,76$ & 3,82 & 8,39 & $43,05-47,10$ \\
\hline V. curassavica (L) & $45,63-56,00$ & $50,63 \pm 0,62$ & 3,09 & 6,09 & $49,35-51,91$ \\
\hline$V$. discolor $(\mathrm{B})$ & $37,50-45,75$ & $40,61 \pm 0,39$ & 1,97 & 4,85 & $39,81-41,41$ \\
\hline V. discolor $(\mathrm{L})$ & $37,13-41,88$ & $40,28 \pm 0,35$ & 1,75 & 4,34 & $39,56-41,00$ \\
\hline V. guazumaefolia (B) & $36,63-48,88$ & $40,13 \pm 0,53$ & 2,66 & 6,62 & $39,03-41,23$ \\
\hline V. guazumaefolia (L) & $40,50-49,25$ & $43,97 \pm 0,46$ & 2,31 & 5,26 & $43,02-44,92$ \\
\hline V. leucocephala $(\mathrm{B})$ & $56,88-70,13$ & $64,32 \pm 0,65$ & 3,24 & 5,03 & $62,99-65,65$ \\
\hline V. leucocephala (L) & $62,50-79,25$ & $69,82 \pm 0,87$ & 4,37 & 6,25 & $68,02-71,62$ \\
\hline V. monosperma $(\mathrm{L})$ & $42,38-52,88$ & $47,38 \pm 0,61$ & 3,05 & 6,44 & $46,12-48,64$ \\
\hline$V$. sessilifolia $(\mathrm{B})$ & $58,53-60,51$ & $59,52 \pm 0,48$ & 2,41 & 4,06 & $58,53-60,51$ \\
\hline V. sessilifolia $(\mathrm{L})$ & $53,88-65,38$ & $59,39 \pm 0,58$ & 2,90 & 4,88 & $58,20-60,58$ \\
\hline V. truncata $(\mathrm{B})$ & $43,88-53,00$ & $48,93 \pm 0,46$ & 2,32 & 4,75 & $47,97-49,89$ \\
\hline V. truncata $(\mathrm{L})$ & $41,25-52,63$ & $46,90 \pm 0,58$ & 2,92 & 6,25 & $45,70-48,10$ \\
\hline V. urticaefolia (B) & $41,13-51,25$ & $46,74 \pm 0,52$ & 2,58 & 5,52 & $45,68-47,80$ \\
\hline V. urticaefolia (L) & $46,50-58,75$ & $52,02 \pm 0,53$ & 2,66 & 5,12 & $50,92-53,12$ \\
\hline
\end{tabular}

Tabela 7. Média aritmética das medidas $(\mu \mathrm{m})$ dos grãos de pólen 3-porados das espécies de Cordiaceae: poro, lúmem e das camadas da exina $(n=10)$. $(\mathrm{B}=$ brevistila; $\mathrm{L}=$ longistila; Compr. = comprimento; Larg. = largura; $\mathrm{P}=$ diâmetro polar; $\mathrm{E}=$ diâmetro equatorial).

Table 7. Arithmetic average of measures $(\mu \mathrm{m})$ of 3-porate pollen grains of Cordiaceae: pore, lumen and exine layers $(n=10)$. ( $\mathrm{B}=$ shortstyle; $\mathrm{L}=$ longstyle; Compr. = length; Larg. = width; $\mathrm{P}=$ polar diameter; $\mathrm{E}=$ equatorial diameter $)$.

\begin{tabular}{|c|c|c|c|c|c|c|c|c|}
\hline \multirow{2}{*}{ Espécies de Varronia } & \multicolumn{2}{|c|}{ Poro } & \multirow{2}{*}{$\frac{\text { Lúmen }}{\text { Faixa de variação }}$} & \multirow{2}{*}{ Exina } & \multirow{2}{*}{ Sexina } & \multirow{2}{*}{ Nexina } & \multirow{2}{*}{ Teto } & \multirow{2}{*}{$\mathrm{P} / \mathrm{E}$} \\
\hline & Compr. & Larg. & & & & & & \\
\hline V. calocephala $(\mathrm{B})$ & 8,56 & 6,54 & $1,17-2,08$ & 4,68 & 2,80 & 1,89 & 1,52 & 0,91 \\
\hline V. calocephala $(\mathrm{L})$ & 7,29 & 6,95 & $1,42-3,42$ & 4,92 & 2,53 & 2,44 & 1,07 & 0,95 \\
\hline V. curassavica (B) & 6,46 & 6,64 & $1,83-4,58$ & 4,72 & 3,04 & 1,09 & 1,29 & 0,91 \\
\hline V. curassavica $(\mathrm{L})$ & 7,13 & 7,18 & $2,08-5,25$ & 5,60 & 3,64 & 1,93 & 1,25 & 0,92 \\
\hline V. discolor (B) & 6,00 & 5,04 & $1,75-3,58$ & 3,92 & 2,35 & 1,57 & 1,75 & 0,93 \\
\hline V. discolor $(\mathrm{L})$ & 5,65 & 5,51 & $1,58-5,42$ & 4,27 & 2,61 & 1,66 & 1,95 & 0,94 \\
\hline V. guazumaefolia (B) & 6,39 & 5,71 & $1,00-3,50$ & 4,49 & 2,73 & 1,77 & 1,34 & 0,91 \\
\hline V. guazumaefolia (L) & 7,24 & 6,85 & $1,58-4,08$ & 5,40 & 3,13 & 2,27 & 1,40 & 0,91 \\
\hline V. leucocephala (B) & 7,15 & 6,61 & $2,33-6,08$ & 5,14 & 3,38 & 1,76 & 1,35 & 0,93 \\
\hline V. leucocephala (L) & 7,78 & 6,26 & $1,42-4,75$ & 5,35 & 2,93 & 2,42 & 2,19 & 0,92 \\
\hline$V$. monosperma $(\mathrm{B})$ & 6,15 & 5,83 & $1,50-4,08$ & 4,36 & 2,29 & 2,08 & 1,66 & 0,95 \\
\hline V. monosperma (L) & 5,15 & 5,14 & $1,00-2,75$ & 4,89 & 3,01 & 1,88 & 1,52 & 0,88 \\
\hline V. sessilifolia $(\mathrm{B})$ & 9,49 & 8,24 & $1,50-3,42$ & 4,83 & 2,63 & 2,20 & 1,07 & 0,92 \\
\hline V. sessilifolia $(\mathrm{L})$ & 9,98 & 7,08 & $1,50-5,75$ & 4,61 & 2,60 & 2,01 & 1,52 & 0,92 \\
\hline V. truncata $(\mathrm{B})$ & 6,05 & 4,78 & $1,67-3,08$ & 5,07 & 2,80 & 2,27 & 1,07 & 0,89 \\
\hline V. truncata $(\mathrm{L})$ & 4,69 & 4,49 & $1,25-3,25$ & 4,13 & 2,23 & 1,90 & 0,99 & 0,89 \\
\hline V. urticaefolia (B) & 5,55 & 5,71 & $1,34-5,16$ & 5,48 & 3,24 & 2,24 & 1,25 & 0,94 \\
\hline V. urticaefolia (L) & 6,79 & 6,81 & $1,50-3,83$ & 4,90 & 3,05 & 1,85 & 1,27 & 0,90 \\
\hline
\end{tabular}


Tabela 8. Média aritmética das medidas $(\mu \mathrm{m})$ dos grãos de pólen 3-porados dos materiais de comparação de Cordiaceae $(n=10)$. ( $\mathrm{B}=$ brevistila; $\mathrm{L}=$ longistila).

Table 8. Measure $(\mu \mathrm{m})$ of arithmetic average of 3-porate pollen grains obtained from species of comparison of Cordiaceae $(n=10)$. (B = shortstyle; $\mathrm{L}=$ longstyle).

\begin{tabular}{|c|c|c|c|c|}
\hline \multirow[b]{2}{*}{ Espécies de Varronia/coletores } & \multicolumn{2}{|c|}{ Vista equatorial } & \multirow{2}{*}{$\begin{array}{c}\text { Vista polar } \\
\text { Diâmetro } \\
\text { equatorial }\end{array}$} & \multirow[b]{2}{*}{$\mathrm{P} / \mathrm{E}$} \\
\hline & $\begin{array}{c}\text { Diâmetro } \\
\text { polar }\end{array}$ & $\begin{array}{l}\text { Diâmetro } \\
\text { equatorial }\end{array}$ & & \\
\hline \multicolumn{5}{|l|}{ V. curassavica (B) } \\
\hline Spina 38 & 50,44 & 55,36 & 52,18 & 0,91 \\
\hline Spina 391 & 48,15 & 54,55 & 53,25 & 0,88 \\
\hline Taroda s.n. (SJRP20333) & 46,67 & 53,12 & 51,25 & 0,88 \\
\hline \multicolumn{5}{|l|}{ V. curassavica $(\mathrm{L})$} \\
\hline Hoffmann et al. 62 & 47,38 & 54,41 & 51,41 & 0,87 \\
\hline Hoffmann et al. 66 & 49,53 & 53,39 & 51,50 & 0,93 \\
\hline Leitão Filho et al. 34652 & 43,08 & 48,99 & 47,23 & 0,88 \\
\hline Souza et al. 3584 & 45,78 & 51,28 & 48,13 & 0,89 \\
\hline Spina 348 & 53,04 & 57,90 & 50,31 & 0,92 \\
\hline \multicolumn{5}{|l|}{ V. discolor $(\mathrm{B})$} \\
\hline Bertoncini \& Cazetta-Neto 978 & 47,94 & 52,58 & 50,75 & 0,91 \\
\hline Melzi \& Avelisso s.n. (SJRP13926) & 43,28 & 47,07 & 45,15 & 0,92 \\
\hline Silva 2845 & 50,28 & 52,93 & 50,83 & 0,95 \\
\hline \multicolumn{5}{|l|}{ V. monosperma (B) } \\
\hline Bernacci 34149 & 53,87 & 56,98 & 54,10 & 0,95 \\
\hline Assis et al. 300 & 45,34 & 50,23 & 46,19 & 0,90 \\
\hline Figueira \& Souza s.n. (SJRP25847) & 51,70 & 55,50 & 54,54 & 0,93 \\
\hline \multicolumn{5}{|l|}{ V. sessilifolia (B) } \\
\hline Lad Netto 217 & 48,93 & 51,51 & 48,01 & 0,95 \\
\hline Loefgren 289 & 51,74 & 57,53 & 56,80 & 0,90 \\
\hline \multicolumn{5}{|l|}{ V. urticifolia (B) } \\
\hline Souza 9472 & 47,40 & 52,71 & 50,29 & 0,90 \\
\hline Souza et al. 11203 & 46,06 & 48,41 & 47,88 & 0,95 \\
\hline
\end{tabular}

Tabela 9. Coeficientes de correlação de Pearson e Kendall entre as variáveis métricas dos grãos de pólen 3-porados e os dois primeiros eixos da ordenação pela PCA para as espécies estudadas de Cordiaceae.

Table 9. Pearson and Kendall correlation coefficients of metric variables between 3-porate pollen grains and the first two axis of PCA ordination, family Cordiaceae.

\begin{tabular}{llcc}
\hline \multirow{2}{*}{ Variáveis } & \multicolumn{2}{c}{ Componentes Principais } \\
\cline { 3 - 4 } & & Eixo 1 & Eixo 2 \\
\hline DPVE & (diâmetro polar em vista equatorial) & $-0,961$ & $+0,225$ \\
DEVE & (diâmetro equatorial em vista equatorial) & $-0,953$ & $+0,254$ \\
DEVP & (diâmetro equatorial em vista polar) & $-0,959$ & $+0,263$ \\
PCOM & (comprimento do poro) & $-0,808$ & $-0,511$ \\
PLAR & (largura do poro) & $-0,736$ & $-0,599$ \\
EXIN & (espessura da exina) & $-0,499$ & $+0,030$ \\
SEXI & (espessura da sexina) & $-0,243$ & $-0,169$ \\
NEXI & (espessura da nexina) & $-0,438$ & $+0,406$ \\
TETO & (espessura do teto) & $+0,001$ & $-0,037$ \\
\hline
\end{tabular}



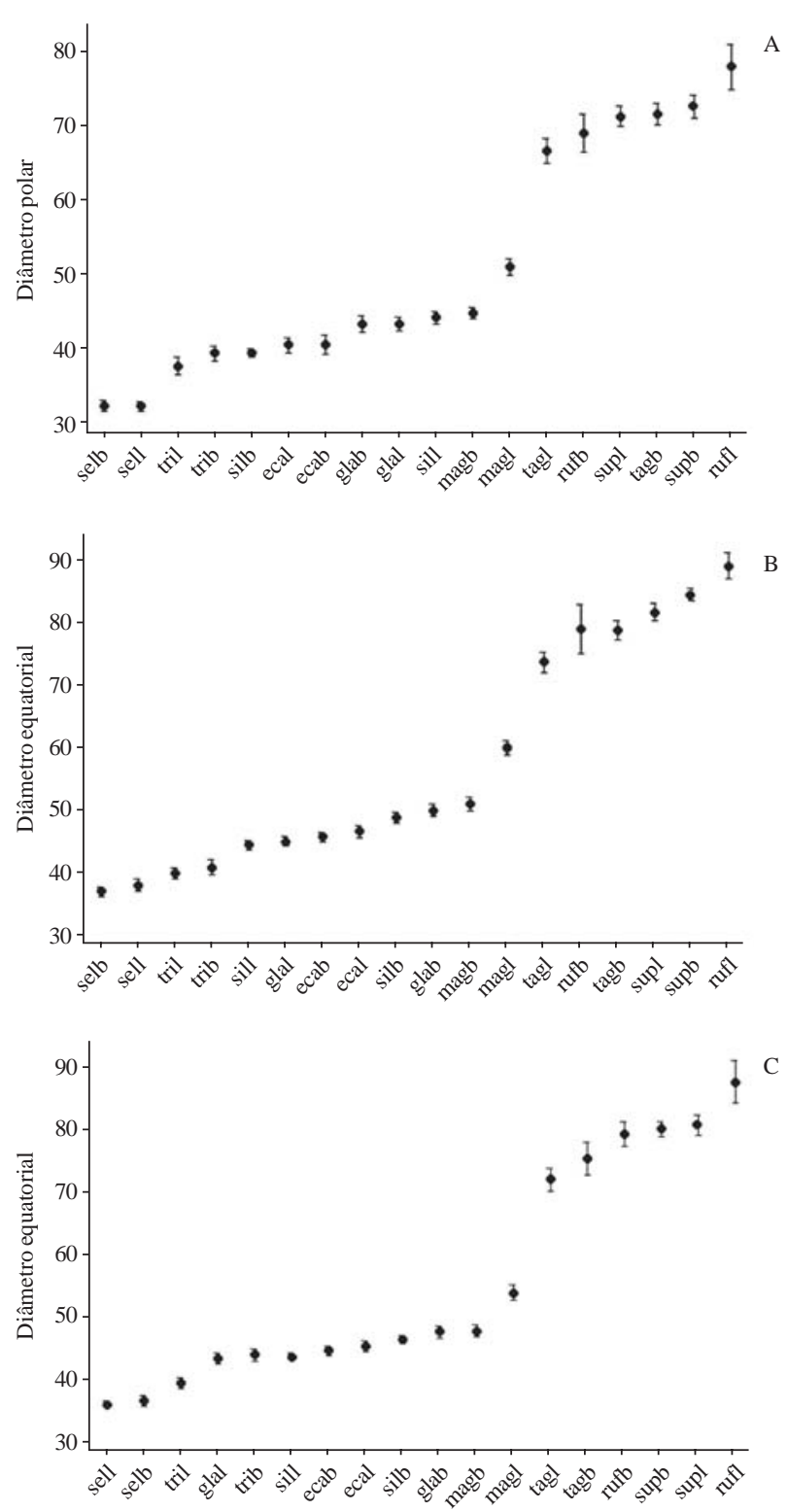

Figura 61. Representação gráfica do intervalo de confiança da média a 95\% dos grãos de pólen 3-colpados de espécies de Cordiaceae. A. Diâmetro polar em vista equatorial. B. Diâmetro equatorial em vista equatorial. C. Diâmetro equatorial em vista polar. Os limites superiores e inferiores representam o intervalo de confiança; os círculos medianos representam a média aritmética. Os valores são dados em $\mu \mathrm{m}$. (ecab = Cordia ecalyculata brevistila; ecal $=C$. ecalyculata longistila; glab $=$ C. glabrata brevistila; glal $=C$. glabrata longistila; magb $=$ C. magnolifolia brevistila; $\mathrm{magl}=$ C. magnolifolia longistila; rufb $=C$. rufescens brevistila; rufl $=C$. rufescens longistila; $\mathrm{selb}=$ C. sellowiana brevistila; sell $=$ C. sellowiana longistila; silb $=C$. silvestris brevistila; sill $=C$. silvestris longistila; supb $=C$. superba brevistila; supl $=C$. superba longistila; $\operatorname{tagb}=C$. taguahyensis brevistila; tagl $=C$. taguahyensis longistila; trib $=C$. trichotoma brevistila; tril $=$ C. trichotoma longistila). e V. calocephala brevistila, apresentaram grãos de pólen com maior valor de comprimento de poro o que conferiu ordenação ao lado inferior do eixo 2, em contraste $V$. truncata longistila, $V$. monosperma longistila e $V$. urticifolia brevistila apresentaram grãos de pólen com os menores valores para esta variável.

\section{Discussão}

Poucos autores analisaram de forma criteriosa as variações dos grãos de pólen da família Cordiaceae, principalmente, levando-se em consideração a heterostilia. Dessa forma, o dimorfismo polínico em espécies heterostílicas foi citado apenas quando se descrevem espécies isoladas (Taroda 1984, Nowicke \& Miller 1990) ou algumas vezes, nos trabalhos de biologia floral (Opler et al. 1975, Percival 1974, Gibbs \& Taroda 1983).

Grãos de pólen 3-colporados, com colpos longos e endoabertura lalongadas foram também encontrados por Barth \& Silva (1963), Nowicke \& Ridgway (1973), Miranda et al. (1980), Taroda (1984), Palacios-Chávez \& Quiroz-Garcia (1985), Moncada \& Herrera (1988), Nowicke \& Miller (1990), Scheel \& Barth (1995), Scheel et al. (1996) e Melhem et al. (2003) ao estudarem o gênero Cordia.

Os grãos de pólen de C. sellowiana foram estudados por Barth \& Silva (1963), Taroda (1984), Scheel \& Barth (1995) e Scheel et al. (1996). Com relação ao presente estudo, houve divergência somente em relação à forma dos grãos de pólen, que foram descritos como oblato-esferoidais (Barth \& Silva 1963, Scheel et al. 1996), prolato-esferoidais (Taroda 1984) e suboblatos (Scheel \& Barth 1995). Os dados referentes aos vários espécimes aqui estudados mostram grãos de pólen

Figure 61. Representation of confidence interval of mean in $95 \%$ of 3-colpate pollen grains of Cordiaceae. A. Polar diameter in equatorial view. B. Equatorial diameter in equatorial view. C. Equatorial diameter in polar view. The higher and lower boundaries showing the confidence interval; the average circle showing the arithmetic mean. The values are in $\mu \mathrm{m}$. (ecab = Cordia ecalyculata shortstyle; ecal $=C$. ecalyculata longstyle; glab $=C$. glabrata shortstyle; glal $=C$. glabrata longstyle; magb $=C$. magnolifolia shortstyle; $\mathrm{magl}=C$. magnolifolia longstyle; rufb $=C$. rufescens shortstyle; rufl $=$ C. rufescens longstyle; selb $=C$. sellowiana shortstyle; sell $=C$. sellowiana longstyle; silb $=C$. silvestris shortstyle; sill $=C$. silvestris longstyle; supb $=C$. superba shortstyle; supl $=C$. superba longstyle; tagb $=C$. taguahyensis shortstyle; tagl $=C$. taguahyensis longstyle; trib $=C$. trichotoma shortstyle; tril $=$ C. trichotoma longstyle). 
variando de suboblatos a oblato-esferoidais, corroborando os trabalhos de Barth \& Silva (1963), Scheel \& Barth (1995) e Scheel et al. (1996).
Quando os dados aqui apresentados para $C$. ecalyculata foram confrontados com os de outros autores que também estudaram palinologicamente a mesma

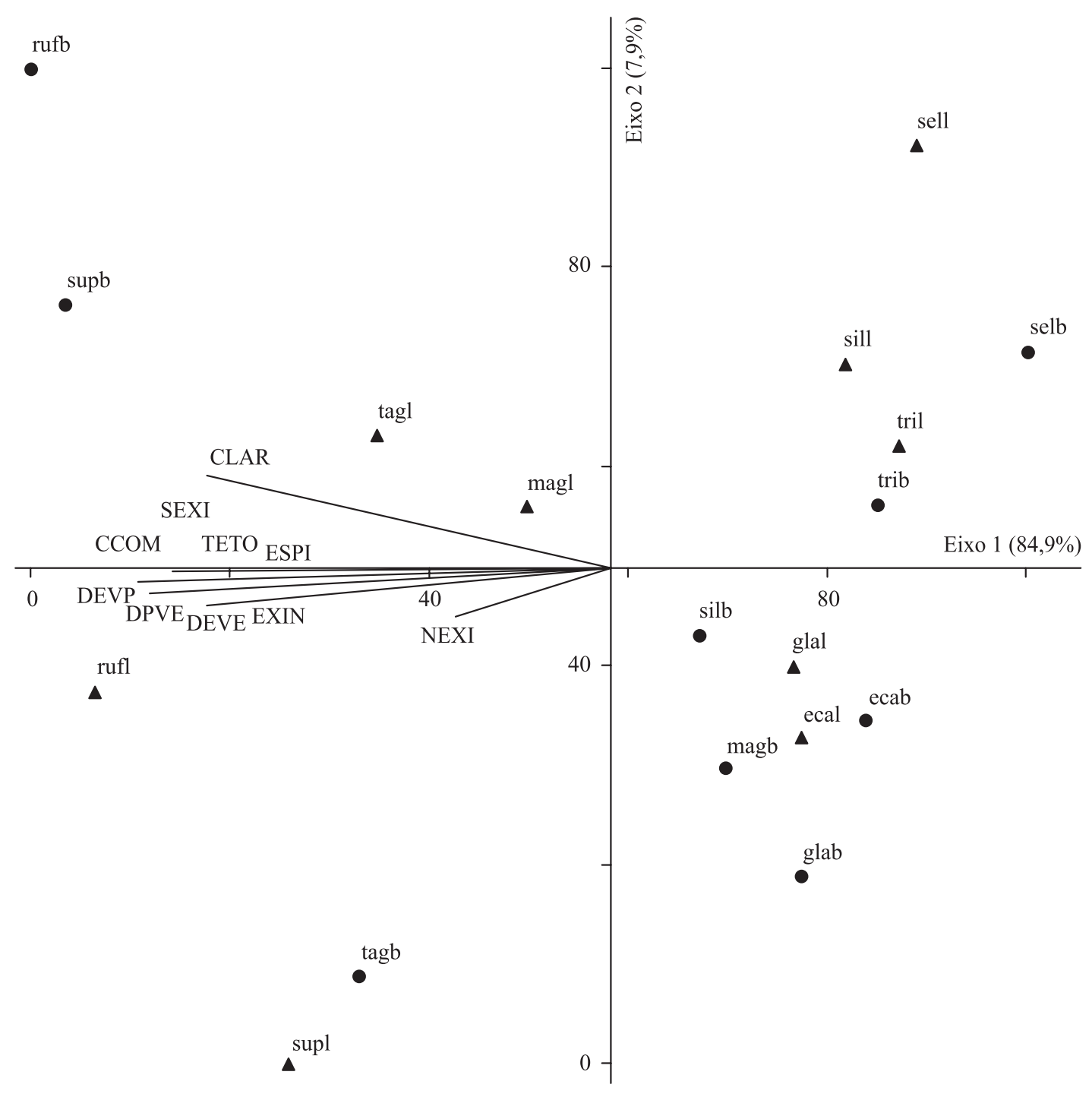

Figura 62. Ordenação, pela PCA, das espécies de Cordiaceae, em função das variáveis métricas dos grãos de pólen 3-colpados. $($ ecab $=$ Cordia ecalyculata brevistila; ecal $=C$. ecalyculata longistila; glab $=C$. glabrata brevistila; glal $=C$. glabrata longistila; magb $=C$. magnolifolia brevistila; $\mathrm{magl}=C$. magnolifolia longistila; rufb $=C$. rufescens brevistila; rufl $=C$. rufescens longistila; selb $=C$. sellowiana brevistila; sell $=C$. sellowiana longistila; silb $=C$. silvestris brevistila; sill $=C$. silvestris longistila; supb $=$ C. superba brevistila; supl $=$ C. superba longistila; tagb $=C$. taguahyensis brevistila; tagl $=C$. taguahyensis longistila; trib $=C$. trichotoma brevistila; tril $=C$. trichotoma longistila; $\bullet=$ brevistila; $\boldsymbol{\Lambda}=$ longistila).

Figure 62. Biplot of PCA for Cordiaceae species, of 3-colpate pollen grains metric variables. (ecab = Cordia ecalyculata shortstyle; ecal $=$ C. e calyculata longstyle; glab $=$ C. glabrata shortstyle; glal $=$ C. glabrata longstyle; magb $=$ C. magnolifolia shortstyle; $\mathrm{magl}=$ C. magnolifolia longstyle; $\mathrm{rufb}=$ C. rufescens shortstyle; rufl $=$ C. rufescens longstyle; $\mathrm{selb}=$ C. sellowiana shortstyle; sell $=C$. sellowiana longstyle; silb $=C$. silvestris shortstyle; sill $=C$. silvestris longstyle; supb $=C$. superba shortstyle; supl $=C$. superba longstyle; tagb $=C$. taguahyensis shortstyle; tagl $=$ C. taguahyensis longstyle; trib $=$ C. trichotoma shortstyle; tril $=$ C. trichotoma longstyle; $\bullet=$ brevistila; $\boldsymbol{\Lambda}=$ longistila) . 

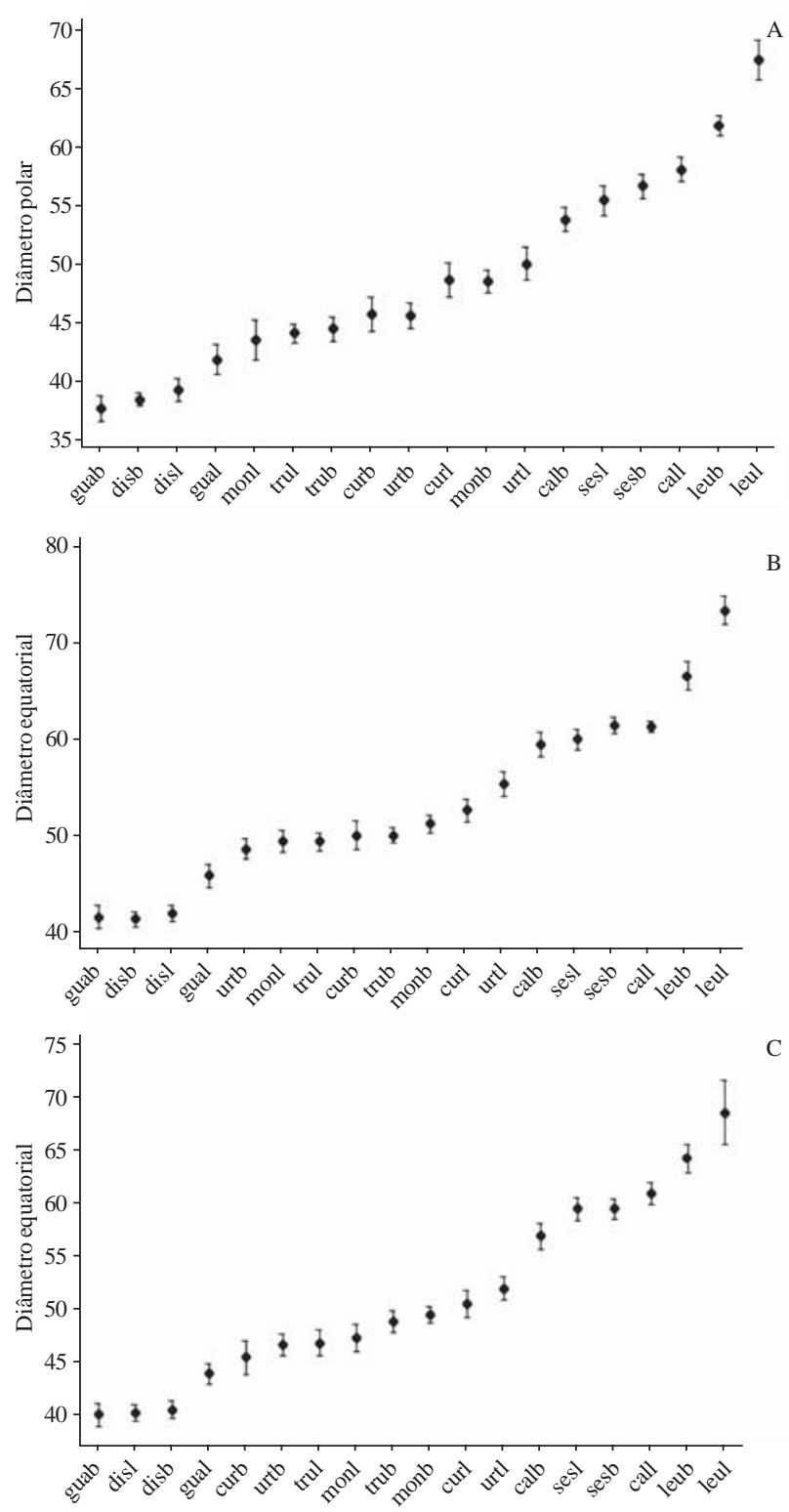

Figura 63. Representação gráfica do intervalo de confiança da média a 95\% dos grãos de pólen 3-porados de espécies de Cordiaceae. A. Diâmetro polar em vista equatorial. B. Diâmetro equatorial em vista equatorial. C. Diâmetro equatorial em vista polar. Os limites superiores e inferiores representam o intervalo de confiança; os círculos medianos representam a média aritmética. Os valores são dados em $\mu \mathrm{m}$. (calb $=$ Varronia calocephala brevistila; call $=V$. calocephala longistila; curb $=V$. curassavica brevistila; curl $=V$. curassavica longistila; disb $=V$. discolor brevistila; disl $=V$. discolor longistila; guab $=V$. guazumaefolia brevistila; gual $=V$. guazumaefolia longistila; leub $=V$. leucocephala brevistila; leul $=V$. leucocephala longistila; monb $=V$. monosperma brevistila; monl $=V$. monosperma longistila; sesb $=V$. sessilifolia brevistila; sesl $=V$. sessilifolia longistila; trub $=V$. truncata brevistila; trul $=V$. truncata longistila; urtb $=V$. urticifolia brevistila; urtl $=V$. urticifolia longistila). espécie (Nowicke \& Ridgway 1973, Scheel \& Barth 1995, Scheel et al. 1996), verificou-se uma concordância em relação ao tipo de abertura (3-colporada) com exceção de Nowicke \& Ridgway (1973) que definiram os grãos de pólen como 3-colporoidados, o que pode ser explicado pela dificuldade de visualização das aberturas.

C. trichotoma foi examinada palinologicamente por Nowicke \& Ridgway (1973), Miranda et al. (1980), Scheel \& Barth (1995) e Scheel et al. (1996). Houve divergência quanto ao tipo de abertura, pois somente Nowicke \& Ridgway (1973) encontraram grãos de pólen 3-colpados, enquanto os demais autores consideram a espécie como 3-colporada, o que está de acordo com o presente trabalho. Quanto à ornamentação da sexina Miranda et al. (1980) descrevem-na como granulada, divergindo de Nowicke \& Ridgway (1973) e Scheel \& Barth (1995) que citaram ornamentação espiculosa. No presente estudo, observou-se que a sexina de $C$. trichotoma é espinhosa confirmando os dados de Scheel et al. (1996). Os valores dos diâmetros dos grãos de pólen nos espécimes aqui medidos são semelhantes aos de Nowicke \& Ridgway (1973) e menores que os dos demais autores. Scheel \& Barth (1995) e Scheel et al. (1996) observaram a presença de grãos de pólen 4-colporados, embora numa proporção maior (47-53\%), quando comparados com este estudo (7\%). Os dados aqui apresentados corroboram os de Gibbs \& Taroda (1983) que estudando heterostilia em Cordia alliodora e $C$. trichotoma encontraram valores de medidas maiores nas flores brevistilas de C. trichotoma.

Taroda (1984), Scheel \& Barth (1995) e Scheel et al. (1996) estudaram $C$. silvestris descrevendo os grãos de pólen da espécie como prolato-esferoidais, suboblatos e oblato-esferoidais, respectivamente. No presente

Figure 63. Graphic representation of confidence interval of mean in $95 \%$ of 3-porate pollen grains of Cordiaceae. A. Polar diameter in equatorial view. B. Equatorial diameter in equatorial view. C. Equatorial diameter in polar view. The higher and lower boundaries showing the confidence interval; the average circle showing the arithmetic mean. The values are in $\mu \mathrm{m}$. (calb $=$ Varronia calocephala shortstyle; call $=V$. calocephala longstyle; curb $=V$. curassavica shortstyle; curl $=V$. curassavica longstyle; disb $=V$. discolor shortstyle; disl $=V$. discolor longstyle; guab $=V$. guazumaefolia shortstyle; gual $=V$. guazumaefolia longstyle; leub $=V$. leucocephala shortstyle; leul $=V$. leucocephala longstyle; $\operatorname{monb}=V$. monosperma shortstyle; monl $=V$. monosperma longstyle; $\mathrm{sesb}=V$. sessilifolia shortstyle; sesl $=V$. sessilifolia longstyle; trub $=V$. truncata shortstyle; trul $=V$. truncata longstyle; urtb $=V$. urticifolia shortstyle; urtl $=$ V. urticifolia longstyle) . 
trabalho observou-se grãos de pólen suboblatos a oblatoesferoidais, com diâmetros significativamente maiores que os indicados por Taroda (1984) e semelhantes aos citados por Scheel \& Barth (1995) e Scheel et al. (1996).
Nowicke \& Miller (1990) descreveram os grãos de pólen de $C$. rufescens e $C$. taguahyensis com forma variando de oblato-esferoidal a esferoidal, 3-colporoidados (em ambas espécies), e com ornamentação espiculada

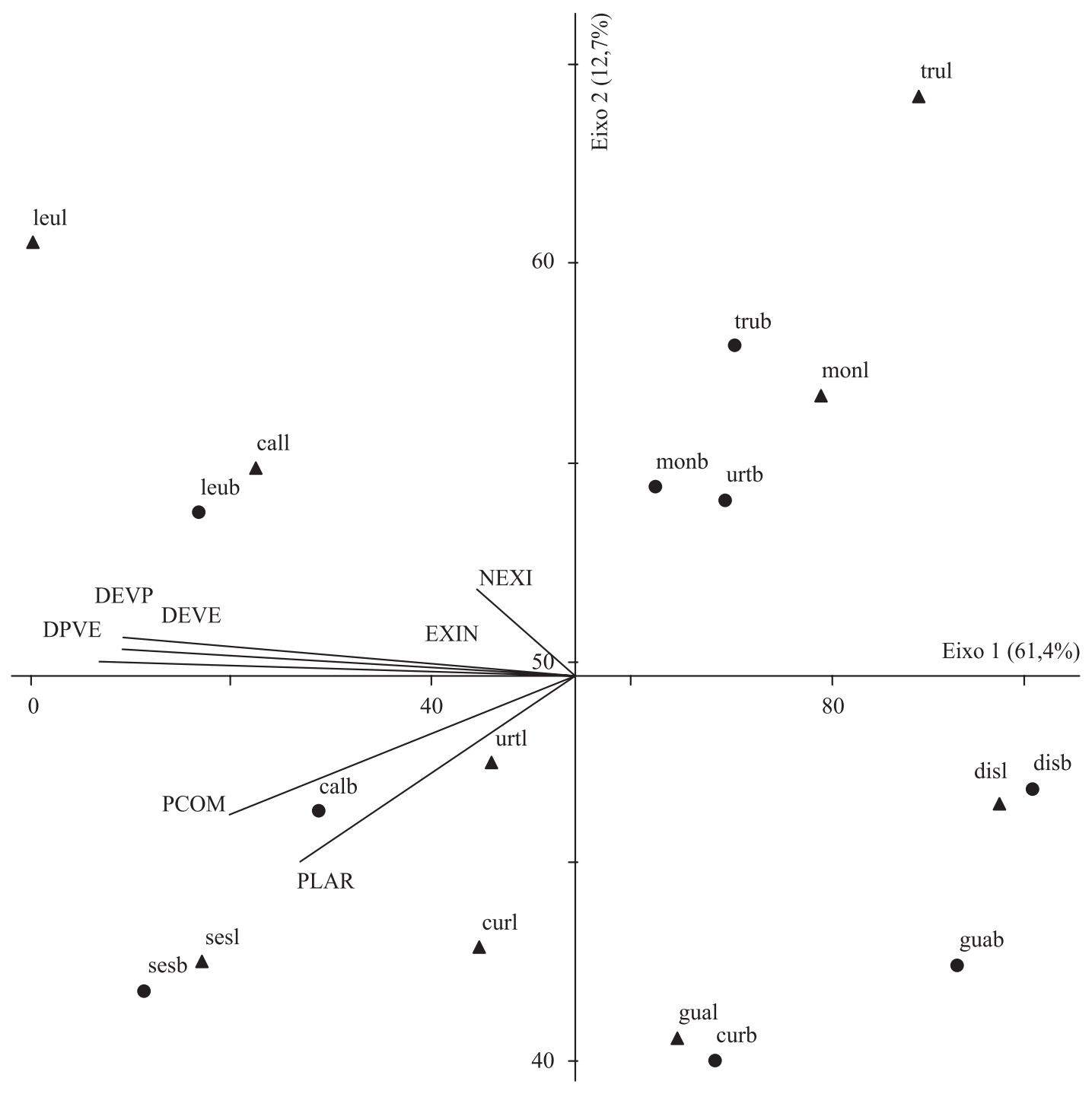

Figura 64. Ordenação, pela PCA, das espécies de Cordiaceae, em função das variáveis métricas dos grãos de pólen 3-porados. $(\mathrm{calb}=$ Varronia calocephala brevistila; call $=V$. calocephala longistila $;$ curb $=V$. curassavica brevistila $;$ curl $=V$. curassavica longistila; disb $=V$. discolor brevistila; disl $=V$. discolor longistila; guab $=V$. guazumaefolia brevistila; gual $=V$. guazumaefolia longistila; leub $=V$. leucocephala brevistila; leul $=V$. leucocephala longistila; monb $=V$. monosperma brevistila; monl $=V$. monosperma longistila; sesb $=V$. sessilifolia brevistila; sesl $=V$. sessilifolia longistila; trub $=V$. truncata brevistila; trul $=V$. truncata longistila; urtb $=$ V. urticifolia brevistila; urtl $=$ V. urticifolia longistila; $\bullet=$ brevistila; $\mathbf{\Lambda}=$ longistila).

Figure 64. Biplot of PCA for Cordiaceae species, of 3-porate pollen grain metric variables. (calb = Varronia calocephala shortstyle; call $=V$. calocephala longstyle $;$ curb $=V$. curassavica shortstyle $;$ curl $=V$. curassavica longstyle $;$ disb $=V$. discolor shortstyle; disl $=V$. discolor longstyle; guab $=V$. guazumaefolia shortstyle; gual $=V$. guazumaefolia longstyle; leub $=V$. leucocephala shortstyle; leul $=V$. leucocephala longstyle; monb $=V$. monosperma shortstyle; monl $=V$. monosperma longstyle; sesb $=V$. sessilifolia shortstyle; sesl $=V$. sessilifolia longstyle; trub $=V$. truncata shortstyle; trul $=V$. truncata longstyle; urtb $=V$. urticifolia shortstyle; urtl $=$ V. urticifolia longstyle; $\bullet=$ brevistila; $\boldsymbol{\Lambda}=$ longistila). 
(C. rufescens) a clavada ( $C$. taguahyensis). Observou-se aqui, grãos de pólen suboblatos em $C$. rufescens e oblatoesferoidais em C. taguahyensis, 3-colporados e com exina espinhosa.

Os grãos de pólen das flores brevistilas e longistilas de $C$. glabrata foram estudados por Taroda (1984) que os descreveu como prolato-esferoidais e com tamanho maior nas flores brevistilas, fato que também foi observado neste estudo.

C. superba apresenta grãos de pólen 3-colporados, confirmando dados também observados por Melhem et al. (2003). Entretanto, Nowicke \& Miller (1990) mencionam a ocorrência de grãos de pólen 3-colporoidados, talvez devido à difícil visualização da endoabertura. Os dados aqui obtidos, assim como os de Melhem et al. (2003), mostraram, ainda, forma suboblata e exina com ornamentação espículo-verrugosa, sendo estas observações contrárias às de Nowicke \& Miller (1990) que mencionaram grãos de pólen oblato-esferoidais com exina espiculada. As medidas dos diâmetros dos grãos de pólen, obtidas para os espécimes aqui analisados são significativamente maiores que as observadas por Melhem et al. (2003) e Nowicke \& Miller (1990).

Grãos de pólen com medidas de diâmetros maiores em flores brevistilas de $C$. glabrata, $C$. trichotoma, $C$. silvestris e $C$. taguahyensis, e em flores longistilas de C. ecalyculata, C. magnoliifolia, C. sellowiana, $C$. rufescens e $C$. superba confirmaram as afirmações de Ganders (1979), que menciona a ocorrência de pólen com diâmetros maiores tanto em flores brevistilas quanto em flores longistilas, dependendo do táxon analisado.

Taxonomicamente, as espécies aqui estudadas, que apresentaram padrão polínico 3-colporado e exina espinhosa a espículo-verrugosa enquadram-se no gênero Cordia, clado Collococcus, o qual compreende as espécies do Novo Mundo (antiga seção Myxa) e as seis espécies brasileiras que Taroda \& Gibbs (1986) haviam posicionado na seção Superbiflorae (Miller \& Gottschling 2007).

As espécies de Cordia possuem grãos de pólen morfologicamente semelhantes, o que não permite separálas com base nos dados polínicos já que caracteres como o diâmetro dos grãos de pólen e a medida de espessura das camadas da exina são bastante similares. Esta afinidade polínica entre as espécies foi comprovada através da analise de componentes principais, na qual somente os grãos de pólen de C. magnoliifolia longistila, ficaram isolados das demais espécies, por apresentarem valores métricos maiores. Por outro lado, as espécies da antiga seção Superbiflorae (C. rufescens, C. superba e C. taguahyensis) de Taroda \& Gibbs (1986) aparecem isoladas das outras espécies dentro do gênero, pelos maiores valores dos dados quantitativos, apesar dos grãos de pólen de $C$. superba possuírem ornamentação espículo-verrugosa.

Grãos de pólen 3-porados, com poros operculados, exina reticulada, de homo a heterorreticulada, simplesbaculada, com espículos sobre os muros dos retículos encontrados nas espécies do gênero Varronia, foram descritos anteriormente por Nowicke \& Ridgway (1973), Taroda (1984), Palacios-Chavez \& QuirozGarcia (1985), Moncada \& Herrera (1988), Nowicke \& Miller (1990) e Scheel et al. (1996). Sendo que todos estes autores trataram as espécies estudadas como pertencentes ao gênero Cordia.

Para V. curassavica há dados polínicos nos trabalhos de Nowicke \& Ridgway (1973), Taroda (1984), Nowicke \& Miller (1990) e Scheel et al. (1996), havendo divergências entre as suas descrições e também em relação aos dados aqui obtidos. Nowicke \& Ridgway (1973) citaram para a espécie grãos de pólen esferoidais, Taroda (1984) os descreveu como prolato-esferoidais, já Nowicke \& Miller (1990) e Scheel et al. (1996), como oblato-esferoidais. No presente estudo os grãos de pólen de V. curassavica se caracterizaram por serem oblatoesferoidais, corroborando os dados de Nowicke \& Miller (1990) e Scheel et al. (1996) quanto à forma.

Segundo Taroda (1984) os grãos de pólen de $V$. calocephala longistila, $V$. discolor brevistila, $V$. guazumaefolia brevistila e $V$. truncata longistila apresentaram forma prolato-esferoidais com exceção de $V$. monosperma longistila, que apresentou grãos de pólen oblato-esferoidais. O presente trabalho difere do de Taroda (1984), pois os espécimes aqui estudados apresentaram grãos de pólen oblato-esferoidais, com exceção de $V$. monosperma longistila, que apresentou grãos de pólen suboblatos.

Para Nowicke \& Miller (1990) as aberturas poradas raramente aparecem em Boraginaceae (incluindo Cordiaceae sensu Jonhston (1950)), mas os autores não esclarecem se o poro presente nos grãos de pólen das espécies do gênero surgiu devido a uma diminuição gradual de um colpo ou se este representa uma endoabertura com ausência de colpo.

As espécies com grãos de pólen 3-porado e exina reticulada (V. calocephala, V. curassavica, V. discolor, $V$. guazumaefolia, $V$. leucocephala, $V$. monosperma, $V$. sessilifolia, V. truncata e V. urticifolia) pertencem ao gênero Varronia descrito por Taroda (1984) como subgênero de Cordia, o qual posteriormente com base em dados moleculares foi reestabelecido por Miller \& Gottschling (2007), corroborando o trabalho de Borhidi et al. (1988). 
A análise de componentes principais incluindo essas espécies mostrou $V$. sessilifolia brevistila e longistila e $V$. curassavica longistila associadas às maiores medidas de largura do poro. O mesmo ocorreu com V. calocephala longistila e $V$. leucocephala longistila, que se associaram as maiores medidas de espessura da nexina. Portanto, apesar da grande similaridade entre os grãos de pólen do subgênero, essas características permitem separar algumas espécies.

Entre todas as espécies cujo pólen foi analisado, 11 delas (C. glabrata, C. trichotoma, C. ecalyculata, $C$. magnoliifolia, C. sellowiana, V. calocephala, $V$. curassavica, V. discolor, V. guazumaefolia, V. leucocephala e $V$. monosperma) apresentam maior espessura de exina nos grãos de pólen das flores longistilas, enquanto nas outras sete $(C$. silvestris, $C$. rufescens, $C$. superba, $C$. taguahyensis, $V$. sessilifolia, $V$. truncata e $V$. urticifolia), os grãos de pólen das flores brevistilas é que apresentam exina com maior espessura.

Após analisar a morfologia polínica de Cordiaceae neste estudo e compará-la com a taxonomia da família, verifica-se que as diferenças dos tipos polínicos coincidem com a delimitação taxonômica proposta primeiramente por Borhidi et al. (1988), e recentemente por Gottschling et al. (2001), Gottschling et al.(2005) e Miller \& Gottschling (2007), sustentando com dados polínicos (abertura e ornamentação da exina) a separação dos gêneros Cordia e Varronia.

Agradecimentos - À Dra. Neusa Taroda Ranga, pela listagem dos táxons ocorrentes no Estado de São Paulo. Ao Dr. Fábio de Barros, pela leitura do manuscrito inicial. À Capes (Coordenadoria de Aperfeiçoamento de Pessoal de Nível Superior), pela bolsa de mestrado concedida ao primeiro autor.

\section{Referências bibliográficas}

BARRET, S.C.H. 1990. The evolution and adaptative significance of heterostyly. Tree 5:144-149.

BARROSO, G.M., PEIXOTO, A.L., ICHASO, C.L., COSTA, C.G., GUIMARÃES, E.F. \& LIMA, H.C. 1986. Sistemática de Angiospermas do Brasil. v.3, Universidade Federal de Viçosa, Imprensa Universitária, Viçosa.

BARTH, O.M. \& SILVA, S.A.F. 1963. Catálogo sistemático dos pólens da plantas arbóreas do Brasil Meridional. IV - Cunoniaceae, Rosaceae e Connaraceae. Memórias do Instituto Oswaldo Cruz 61:411-427.

BARTH, O.M. \& MELHEM, T.S. 1988. Glossário ilustrado de palinologia. Editora da Universidade Estadual de Campinas, Campinas.

BORHIDI, A., GONDÁR, E. \& OROSZ-KOVÁCS, Zs. 1988. The reconsideration of the genus Cordia. Acta Botanica Hungarica 34:375-423.
CAVALHEIRO, L., PERALTA, D.F. \& FURLAN, A. 2003. Flórula fanerogâmica da planície litorânea de Picinguaba, Ubatuba, SP, Brasil: Boraginaceae. Hoehnea 30:173-179.

ERDTMAN, G. 1952. Pollen morphology and plant taxonomy - Angiosperms. Almqvist \& Wiksell, Stockholm.

GANDERS, F.R. 1979. The biology of heterostyly. New Zealand Journal of Botany 17:607-635.

GIBBS, P. 1990. Self-incompatibility in flowering plants: a neotropical perspective. Revista Brasileira de Botânica 13:125-136.

GIBBS, P. \& TARODA, N. 1983. Heterostyly in the Cordia alliodora-C. trichotoma complex in Brazil. Revista Brasileira de Botânica 6:1-10.

GOTTSCHLING, M., HILGER, H.H., WOLF, M. \& DIANE, N. 2001. Secondary structure of the ITS1 transcript and its application in a reconstruction of the phylogeny of Boraginales. Plant Biology 3:629-636.

GOTTSCHLING, M. MILLER, J.S., WEIGEND, M. \& HILGER, H.H. 2005. Congruence of a phylogeny of Cordiaceae (Boraginales) inferred from ITS1 sequence data with morphology, ecology and biogeography. Annals of the Missouri Botanical Garden 92:425-437.

HOLMGREN, P.K. \& HOLMGREN, N.H. 1998 (onwards). Online edition of Index Herbariorum. http://sciweb. nybg.org/science2/IndexHerbariorum.asp (acesso em 05/11/2004).

JOHNSTON, I.M. 1950. Studies in the Boraginaceae 19. Cordia sect. Gerascanthus in Mexico and Central America. Journal of the Arnold Arboretum 31:179-187.

JUDD, W.S., CAMPBELL, C.S., KELLOGG, E.A. \& STEVENS, P.F. 1999. Plant systematics: a phylogenetic approach. Sinauer Associates, Sunderland.

JUNG-MENDAÇOLLI, S.L. \& MELHEM, T.S. 1995. Grãos de pólen de espécies heterostílicas de Rubiaceae. Revista Brasileira de Botânica 18:61-63.

MCCUNE, B. \& MEFFORD, M.J. 1999. PC-ORD. Multivariate analysis of ecological data, version 4.10. MjM Software Design, Oregon.

MELHEM, T.S. \& MATOS, M.E.R. 1972. Variabilidade de forma dos grãos de pólen de Eriope crassipes Benth. Labiatae. Hoehnea 2:1-10.

MELHEM, T.S., CRUZ-BARROS, M.A.V., CORREAA, A.M.S., MAKINO-WATANABE, H., SILVESTRECAPELATO, M.S.F. \& GONÇALVES-ESTEVES, V.L. 2003. Variabilidade polínica em plantas de Campos do Jordão (São Paulo, Brasil). Boletim do Instituto de Botânica de São Paulo 16:1-104.

MILLER, J.S. 2001. New Boraginaceae from tropical America 4: Three nem species of Cordia from South America. Novon 11:421-428.

MILLER, J.S. 2007. New Boraginales from Tropical America 5: New names and typifications for Neotropical species of Cordia and Varronia. Novon 17:372-375. 
MILLER, J.S. \& GOTTSCHLING, M. 2007. Generic classification in the Cordiaceae (Boraginales): resurrection of the genus Varronia P. Br. Taxon 56:163-169.

MINITAB FOR WINDOWS [MINITAB-INC, USA]. 2003. Versão 10.3 .

MIRANDA, M.M.B., CAVALCANTE, M.P.P. \& GODIM, M.E.R. 1980. Pólen das plantas silvestres do Ceará VI. Litoral. Famílias Anacardiaceae, Annonaceae, Boraginaceae, Combretaceae, Sterculiaceae, Malpighiaceae, Polygonaceae, Rutaceae e Turneraceae. Revista Brasileira de Farmácia 61:21-28.

MONCADA, M. \& HERRERA, P.O. 1988. La palinología del género Cordia (Angiospermae: Boraginaceae) en Cuba. Acta Botanica Cubana 58:1-10.

NOWICKE, J.W. \& RIDGWAY, J.E. 1973. Polen studies in the genus Cordia (Boraginaceae). American Journal of Botany 60:584-591.

NOWICKE, J.W. \& MILLER, J.S. 1990. Pollen morphology of the Cordioideae (Boraginaceae). Auxemma, Cordia and Patagonula. Plant Systematics and Evolution 5:103-121.

OPLER, P.A., BAKER, H.G. \& FRANKIE, G.W. 1975. Reprodutive biology of some Costa Rican Cordia species (Boraginaceae). Biotropica 7:234-247.

PALACIOS-CHÁVEZ, R. \& QUIROZ-GARCIA, D.L. 1985. Catálogo palinológico para la flora de Veracruz. Família Boraginaceae, género Cordia. Biotica 10:1-113.

PERCIVAL, M. 1974. Floral ecology of coastal scrub in southeast Jamaica. Biotropica 6:104-129.

PUNT, W., HOEN, P.P., BLACKMORE, S., NILSSON, S. \& LE THOMAS, A. 2007. Glossary of pollen and spore terminology. Review of Paleobotany and Palynology 143:1-81.
SALGADO-LABOURIAU, M.L. 1973. Contribuição à palinologia dos cerrados. Academia Brasileira de Ciências, Rio de Janeiro.

SALGADO-LABOURIAU, M.L., VANZOLINI, P.E. \& MELHEM, T.S. 1965. Variation of polar axes and equatorial diameters in pollen grains of two species of Cassia. Grana 6:166-176.

SCHEEL, R. \& BARTH, O.M. 1995. Catálogo sistemático do pólen das plantas arbóreas do Brasil meridional. XXIX: Boraginaceae. Revista Brasileira de Biologia 55:769-776.

SCHEEL, R., YBERT, J-P. \& BARTH, O.M. 1996. Pollen morphology of the Boraginaceae from Santa Catarina State (southern Brazil), with comments on the taxonomy of the family. Grana 35:138-153.

SHEPHERD, G.J. 1996. Fitopac 1: manual do usuário. Departamento de Botânica, Universidade Estadual de Campinas.

TARODA, N. 1984. Taxonomic studies on Brazilian species of Cordia L. (Boraginaceae). Philosophy Doctor Thesis, University of Saint Andrews, Saint Andrews.

TARODA, N. \& GIBBS, P.E. 1986. Studies on the genus Cordia L. (Boraginaceae) in Brazil. 1. A new infrageneric classification and conspectus. Revista Brasileira de Botânica 9:31-42

TOMLINSON, P.B. 1974. Breeding mechanisms in trees native to tropical Florida - A morphological assessment. Journal of the Arnold Arboretum 55:269-290.

VIEIRA, S. 1981. Introdução à bioestatística. Campus, Rio de Janeiro.

ZAR, J.H. 1984. Biostatistical analysis. Prentice Hall, Englewood Cliffs. 